\title{
O TEOREMA DE HUREWICZ NA TEORIA \\ DE HOMOTOPIA DOS ESPACOS NILPOTENTES
}

PAULO FERREIRA LEITE

\author{
TESE APRESENTADA
}

A0

INSTITUTO DE MATEMATICA E ESTATISTICA

DA

UNIVERSIDADE DE SĀO PAULO

PARA OBTENÇĀO DO GRAU DE DOUTOR

EM

MATEMATTICA

ORIENTADOR:

Prof. Dr. PETER J. HILTON

São Paulo fevereiro de 1979 
Aos meus Pais,

a Marilena, à Cecilia

e à Heloisa. 


\section{ABSTRACT}

The main purpose of this work is the study of the homomorphisms induced in homology by the covering projection in the category of nilpotent spaces.

We work in the context of a Serre class $C$ of nilpotent groups, which is a generalization of the concept of Serre class of abelian groups introduced by Hilton, Mislin and Roitberg ( $\sec [H, M, R],[H, R]_{1}$ ).

Our main result is

THEOREM. Let $\vec{X} \longrightarrow X$ be a regular covering of a nilpotent space $X$. If $X$ is of finite type or $C$ is a complete class then the following conditions are equivalent:

i) $\pi \in C$, where $\pi$ is the group of covering transformations of the covering $\bar{x} \longrightarrow x$;

ii) The homomorphisme induced in homology $\mathrm{H}_{\mathrm{q}} \overrightarrow{\mathrm{X}} \longrightarrow \mathrm{H}_{\mathrm{q}} \mathrm{X}$ by the covering projection is a c-bijetion for every $q$.

The main point in the proof of this theorem is the use of some algebraic results concerning the (co)homology of groups modulo a class C of nilpotent groups, (see theorems 4.1.1;4.1.3).

As applications we get an improvement of an absolute Hurewicz theorem obtained by Hilton and Roitberg (see $[H, R]_{1}$ ) and proved some relative form of the same theorem. 
We also prove a property modulo $C$ of the $\Gamma_{i}{ }^{K}$ groups introduced by J.H.C. Whitehead (see $[\mathrm{W}]_{2}$ ). 


\section{I $N D I C E$}

\section{CAP. I - TEOREMAS DE HUREWICZ CLĀSSICOS}

$\S 0$ - Introdução. . . . . . . . . . . . . 1

$\$ 1$ - Teoremas de Hurewicz. . . . . . . . . . 5

$\$ 2$ - Sequência exata de J,H.C.Whitehead. Adendo ao teorema de Hurewicz. . . . . . . . . . . 15

CAP. II - TEORIA DE SERRE

$\$ 0$ - Introdução. . . . . . . . . . . 19

$\$ 1$ - Classes de Serre de grupos Abelianos. . . . . . 21

$\S 2$ - Teoremas de Hurewicz e de Whitehead mödulo C. . . 26

CAP.III - GRUPOS E AÇÕES NILPOTENTES, CLASSES DE SERRE DE GRUPOS NILPOTENTES E ESPAÇOS NILPOTENTES

\$o - Introdução. . . . . . . . . . . . 33

$\$ 1$ - Grupos e ações nilpotentes. . . . . . . 35

$\$ 2$ - Classes de Serre de grupos nilpotentes. . . . . . 40

$\$ 3$ - Espaços nilpotentes .......... . 46

CAP. IV - ALGUNS RESULTADOS ALGËBRICOS RELATIVOS A (CO) HOMOLOGIA DE GRUPOS MOD. C

$\S 0$ - Introdução. . . . . . . . . . . 51

$\$ 1$ - Homologia ................ 53

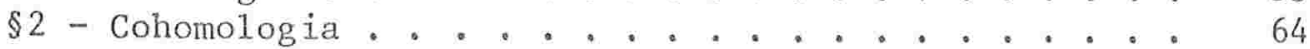

CAP $\vee V$ - HOMOLOGIA MODULO $C$ DE RECOBRIMENTOS DE ESPAÇOS NILPOTENTES

$\S 0$ - Introdução. . . . . . . . . . . .

$\$ 1$ - Espaços de recobrimento: Propriedades homotópicas e de levantamento............. . . .

$\$ 2$ - Espaços de recobrimento: Relação entre os grupos de homologia de um espaço e seu recobrimento. Sequência espectral de um recobrimento. . . . . . 77

$\$ 3$ - Recobrimentos de espaços nilpotentes: homologia mod C 81

$\$ 4$ - Caso relativo. .............. 92

$\$ 5$ - Cohomologia. . . . . . . . . . . . . . . 94

CAP. VI - APLICAÇÕES. O TEOREMA DE HUREWICZ MỎDULO C E A SEQUÊNCIA EXATA DE J.H.C.WHTTEHEAD

$\$ 0$ - Introdução. . . . . . . . . . . . . 97

$\$ 1$ - Aplicaçoes. . . . . . . . . . . . . 99

BIBLIOGRAFIA. . . . . . . . . . . . 107 


\section{INTRODUÇÄO}

Estudamos neste trabalho, no contexto das classes de Serre de grupos nilpotentes, os homomorfismos induzidos em homologia por uma projeção de recobrimento na categoria dos espaços nilpotentes.

Isso nos permitirâ desenvolver, sob um ponto devis ta novo, parte do programa de generalizar para os espaços nilpotentes (e tambëm para os espaços quase-nilpotentes) tệc nicas e resultados de reconhecida importância e utilidadena categoria dos espaços simplesmente conexos. Em particular, pudemos, como aplicações, dar uma versão melhorada do teore ma de Hurewicz módulo C obtido por Hilton e Roitberg em $[H, R]_{1}$; obter, para o caso relativo, uma forma fraca desse mesmo teorema e provar una propriedade mỏdulo $\mathrm{C}$ dos grupos $\Gamma_{i} K$ que figuram numa sequência exata introduzida em 1950 $\left(\operatorname{ver}[\mathrm{W}]_{2}\right)$ por J.H.C. Whitehead.

Antes de passarmos a descrever o trabalho em si,fa remos algumas considerações de caräter geral. 
Atê recentemente, em virtude da importância prätica de resultados como os teoremas de Hurewicz e Whitehead, que só eram conhecidos para espaços simplesmente conexos,os topölogos concentraram seus esforços no estudo desses espaços. A primeira grande generalização que esses teoremas sofreram se deu no princípio da dêcada de 1950 que, diga-sede passagem, foi uma ëpoca de grandes transformaçöes na Topolo gia Algêbrica. Utilizando técnicas que havia desenvolvidoan teriormente em sua tese de doutoramento (veja [S] $]_{1}$ ) sobre a homologia singular dos espaços fibrados e, introduzindo o conceito de classe de grupos abelianos, J.P.Serre pode, em 1953 (conf. $[S]_{2}$ ), dar uma vasta generalização desses teore mas. Permanecia porëm, ainda, a restrição de que os espaços envolvidos deviam ser simplesmente conexos, ou, caso não o fossem, $\pi_{1} X$ devia ser um grupo abeliano e sua ação em todos os outros grupos de homotopia devia ser trivial, como acontece, por exemplo, com os espaços de Hopf. O fato do teorema ser vâlido para essa categoria mais geral do que a dos es paços simplesmente conexos não foi muito enfatizado na ëpoca, mesmo porque, essa categoria não apresentava evidentes vantagens sobre a anterior.

Muito mais recentemente, com a observação feitapor Sullivan em 1970, de que existe na teoria da homotopia uma construção funtorial anäloga à localização em Ảlgebra, uma considerävel quantidade de trabalho foi feita nessa direção 
e se tornou evidente que a categoria dos espaços simplesmen te conexos não ë adequada para o pleno desenvolvimento dessas tecnicas. "This is due principally to the fact that it does not respect function spaces. We know following Milnor, that if $X$ is a (pointed) CW-complex and $W$ a finite (pointed) $C W$-complex, then the function space $X^{W}$ of pointed maps $W \rightarrow X$ has the homotopy type of a CW-complex. However its components will, of course, fail to be 1 -conected even if $X$ is 1 -connected", escrevem Hilton, Misiin e Roitberg (confronte [H,M,R], päg. 62). "Howevex", prosseguem eles, "it turns out that the components of $X^{W}$ are nilpotent if $X$ is nilpotent. Moreover, the category of nilpotent C.W complexes is suitable for homotopy theory (as first pointed out by E. Dror), and for localization techniques."

Muitos matemäticos contribuiram para a popularização dos espaços nilpotentes (confronte definição 3.3 .0 do ca pitulo III). Em particular, E. Dror (veja [D]) provou, em 1971, uma generalização do teorema de Whitehead que inclui esses espaços e Hilton e Roitberg demonstraram mais recente mente (1976) uma versão do teorema de Hurewicz, no caso absoluto, e um teorema de whitehead para essa categoria de es paços (veja, respectivamente, $[H, R]_{1}$ e $[H, R]_{2}$ ). Em $[H, M, R]$, os autores desenvolvem uma teoria de localização para espaços nilpotentes e fazem diversas aplicações.

Neste trabalho, retornamos a ele, apôs a breve di- 
gressão acima, colocamo-nos na categoria dos C.W. complexos e, portanto, a menos que se faça menção explícita emcon trärio, o termo espaço deve ser tomado sempre nessa acepção, isto é, um espaço para nós è sempre um C.W.-complexo. Se al gumas vezes insistimos nesse ponto, no texto, $\vec{e}$ por pura ên fase.

No primeiro capitulo, fazemos um räpido apanhado dos teoremas clässicos de Hurewicz e Whitehead destacando os pontos que irão apresentar maior interesse nos capitulos fu turos. Apenas com o intuito de ilustrar o ponto de vista adotado, apresentamos algumas demonstrações.

Tratamos em seguida, da generalização, dada por Serre, a esses teoremas. Para isso recordamos algumas definições e propriedades das classes de serre de grupos abelia nos. o espirito desse capítulo é o mesmo do anterior.

Concentramos, para comodidade do leitor, no capítulo II , as definições e os enunciados de teoremas que utilizaremos nos capitulos finais do trabalho.

os capitulos quatro, cinco e seis sumarizam nossa contribuição ao estudo dos espaços nilpotentes.

o capitulo IV, de natureza puramente algëbrica, con tëm resultados cruciais para o desenvolvimento do capítulo $V$ que è, em essência, topológico. Abordaremos nele, questõés referentes a (co)homologia dos grupos mödulo uma classe de 
Serre C de grupos nilpotentes.

No capitulo $V$, estudamos homomorfismos induzidos na homo logia, por uma projeção de recobrimento na categoria dos es paços nilpotentes. O principal resultado do capitulo è o teo rema 5.3.4 que, juntamente com seus corolärios fornece, como exemplificamos no capitulo VI, um meio simples e eficien te de generalizar para espaços nilpotentes, resultados conhecidos no caso simplesmente conexo.

No capitulo VI fizemos as aplicações jä mencionadas no inïcio desta introdução.

E com grande prazer que agradecemos a todas pessoas que nos auxiliaram, direta ou indiretamente, na elaboração deste trabalho e, muito particularmente aos professores:

U. Seip, R. Fritsch e Nelson S. Leme, pelas formas diver sas mas igualmente eficazes como nos auxiliaram.

Angelo Barone Neto, nosso antigo orientador, pelo apoio, estímulo e compreensão.

Daciberg Lima Gonçalves pela ajuda decisiva no iníciodes te trabalho.

Elza Furtado Gomide pelo muito que aprendemos em seus se minärios e pela competência com que leu os manuscritos deste trabalho. Suas sugestões foram de grande valia e sem elas o numero de erros, imperfeições e obscuridades seria ain 
da maior.

Peter John Hilton, orientadox deste trabalho, pelo est $\vec{i}$ mulo, orientação e amparo nas horas difïceis. Graças as suas raras qualidades de caräter, o Prof. Hilton soube, apesar de nossas diferentes qualificações profissionais, transformar nosso trabalho conjunto numa atividade recompensadora echeia de prazer.

Finalmente, queremos agradecer a Gisela pelo seu inesperado entusiasmo pela Ålgebra Homológica e ao sr. João Baptista Esteves de O1iveira e RaqueI de Jesus pelo excelente trabalho datilografico. 


\section{CAPITULO I}

\section{TEOREMAS DE HUREWICZ CLASSICOS}

\section{$\S 0$ - INTRODUÇÃO}

Como sabemos, Poincaré, motivado pelo desejo de classifi car variedades, foi o primeiro a empregar, de forma sistemä tica, mëtodos combinatórios no estudo da topologia. Numa sé rie de trabalhos pioneiros, iniciada em 1895 com o artigo Analysis situs (ver $[P]$ ), desenvolveu ele as primeiras jdëias sobre a teoria de homologia e introduziu o conceito de grupo fundamental de um espaço. Era inclusive de seu conhecimen to, embora näo tenha deixado demonstração desse fato, a reIação existente entre $\pi_{1} X$ e $H_{1} X$.

Os grupos de homotopia de oxdem mais alta sö foram, porëm, introduzidos bem mais taxde, entre os anos de 1932 e 1935, com os trabalhos de E. Čech (ver [Če]) e W. Hurewicz (confronte referências abaixo). Tambëm H. Hopf contribuiude forma fundamental para o reconhecimento da importância des- 
ses grupos.

Hurewicz, partindo de una definição adequada dos grupos de homotopia (absolutos e relativos), estudou-os em profundidade em quatro artigos Iamosos (ver [Hu]). En particular, definiu, para cada inteiro $j$, un homomorfismo $\pi_{j} X \stackrel{h}{\longrightarrow} H_{j} X$ e provou que se o espaço $X$ ë simplesmente conexo então o primeiro grupo de homotopia não nulo ê isomorfo ao correspondente grupo de homologia. Provou tambëm um resultado anälogo para o caso relativo.

Sendo esses resultados o tema central do nosso trabalho, cabem aqui alguns comentârios.

E bem sabido que, embora os grupos de homotopia possam ser definidos de uma forma bastante simples e geométrica, o seu cảlculo, salvo em conhecidas exceções, ẻ extremamente di ficil ou mesmo impossivel com os mẻtodos atualmente disponîveis. Em oposição a essa situação, encontram-se os grupos de homologia. De definjção mais elaborada e com menos conteüdo geométrico, são eles, no entanto, mais simplesmente calculäveis.

Somente o fato do teorema de Hurewicz permitir, atravẻs do isomorfismo com o correspondente grupo de homologia, o câl culo do primeiro grupo de homotopia não nulo, jä justifica a sua importância.

Um aspecto marcante no desenvolvimento da teoria clâssi- 
sica de homotopia è a exigência de que os espaços envolvidos sejam simplesmente conexos. O teorema de Hurewicz, que descrevemos acima, não contitui, como ë amplamente sabido, exceção para esse fato.

Nosso objetivo, no próximo parágrafo, é oferecer uma demonstração para o teorema de Hurewicz.

Entre as possîveis demonstrações, escolhemos a que utili za a seqüência espectral de uma fibração. A razão da escotha é dupla: alëm de julgarmos ser essa a melhor demonstração para o teorema, ela ilustrarä a têcnica utilizada nos ca pitulos futuros. Admitimos, portanto, a familiaridade do lei tor com a teoria das sequências espectrais de uma fibração.

Entre as muitas referências existentes para esse toopico, destacamos a fonte original, a tese de doutoramento de J.P. Serre (ver $\left.[S]_{1}\right)$. Esse é um trabalho extraordinariamente lü cido e bem escrito, cuja leitura recomendamos. Outra excelente referência è $[H, W]$.

No parâgrafo 2, damos uma rảpida descrição de uma seqüên cia exata descoberta e estudada por J.H.C.Whitehead ( $\left[W_{2}\right]_{2}$. Essa seqüência exata nos permitirä provar um importante aden do ao teorema de Hurewicz. Provaremos, no capitulo VI, um re sultado envolvendo essa seqüência exata.

Advertimos o leitor para o fato de que, sendo clässico to do o material do capitulo e dispondo-se de excelente biblio 
grafia, fomos, em alguns pontos, bastante concisos. A idẻia geral e apenas ilustrar tëcnicas e fornecer subsidios para os capitulos posteriores. Alẻm das jä mencionadas, poderiamos indicar, como referência, para o capitulo, [M,T]. 
$\S 1$ - TEOREMAS DE HUREWICZ

1.1.0. Recordemos, rapidamente, a definiçäo do homomorfismo $\pi_{x} x \stackrel{h_{n}}{\longrightarrow} H_{n} x$ de Hurewicz.

Seja $\sigma_{n}$ um gerador (existem maneiras naturais de definir esse gerador) de $H_{n}\left(S^{n}\right)$. Se $[f] \epsilon \pi_{n} X$ representa a classe de homotopia da função continua $\mathrm{s}^{\mathrm{n}} \stackrel{f}{\longrightarrow} \mathrm{x}$, definimos

$$
h_{n}[f]=f *\left(\sigma_{n}\right)
$$

No caso relativo, procedemos de maneira anảloga. Um elemento de $\pi_{n}(X, A)$ pode ser interpretado como a classe de homotopia de uma função $\left(E^{n}, S^{n-1}\right) \longrightarrow(X, A)$. Escolhendo-se en tão um gerador $\vec{\sigma}_{n}$ de $H_{n}\left(E^{n}, S^{n-1}\right)$ (por exemplo, atravês do $\underline{i}$ somorfismo $\left.H_{n}\left(E^{n}, S^{n-1}\right) \longrightarrow H_{n-1}\left(S^{n-1}\right)\right)$ definimos

$$
h_{n}[f]=f *\left(\bar{\sigma}_{n}\right)
$$

Prova-se então que $h_{n} \ddot{e}$ um homomorfismo para $n \geq 1(n \geq 2$ no caso relativo).

Com esses preliminares, jā estamos em condições de enunciar o

1.1.1. Teorema de Hurewicz. Seja X um espaço simplesmente co nexo. São equivalentes as condições:
i) $\pi_{j} X=0$
$1 \leq \mathrm{j} \leq \mathrm{n}-1$
ii) $H_{j} X=0$
$1 \leq j \leq n-1$ 
No caso de una das condições acima estar verificada, podemos afirmar que

$$
\text { iii) } \pi_{n} \times \stackrel{h_{n}}{\longrightarrow} H_{n} \times \text { ë um isomorfismo. }
$$

DEMONSTRAÇĀO

Provaremos que i) implica ii) e iii). Dessa implicação decorre imediatamente a equivalência entre i) e ii).

A demonstração é feita por indução sobre n. Provaremos i nicialmente o passo da indução $(n \geq 3)$ e verificamos, posteriormente, que o teorema é välido para $n=2$.

Seja então $x$ un espaço $(n-1)$ conexo. Nossa hipótese de in dução assegura que o teorema è verdadeiro para qualquer espaço $(n-2)$ conexo.

Consideremos agora a fibração

$$
\Omega X \longrightarrow E X \longrightarrow E
$$

onde $\Omega X$ e EX säo, respectivamente, os espaços dos laçosedos caminhos em $x$ e o diagrama comutativo

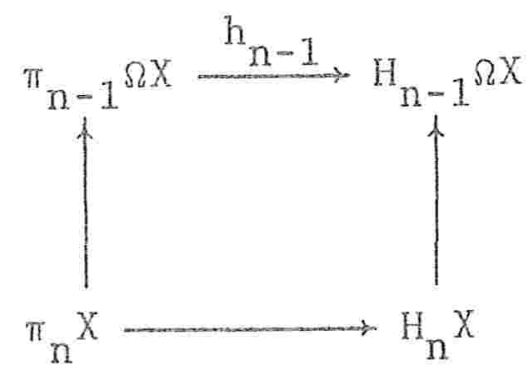

onde as flechas horizontais são os respectivos homomorfismos de Hurewicz, a flecha vertical da esquerda $\ddot{e}$ um isomor.- 
fismo e a flecha vertical da direita $\vec{e}$ a transgressão (cuja existência estä assegurada pela hipótese de indução). Uma vez que a hipötese de indução nos garante que

$$
\pi_{n-1} \Omega X \stackrel{h_{n-1}}{\longrightarrow} H_{n-1} \Omega X
$$

é um isomorfismo, basta que se prove que a transgressão tam bẻm ë, nesse caso, um isomorfismo e teremos a tese.

Utilizaremos para isso a seqüência espectral da fibração $\Omega \mathrm{X} \longrightarrow \mathrm{EX} \longrightarrow \mathrm{X}$

Observemos, inicialmente, o diagrama abaixo, extraidodes sa seqüência espectral:

$$
\begin{aligned}
& \mathrm{H}_{\mathrm{n}-1} \Omega \mathrm{X}=\mathrm{E}_{0, \mathrm{n}-1}^{2} \rightarrow \mathrm{E}_{0, \mathrm{n}-1}^{3} \rightarrow \rightarrow \cdots \rightarrow \mathrm{E}_{0, \mathrm{n}-1}^{\mathrm{n}} \rightarrow \mathrm{E}_{0, \mathrm{n}-1}^{\mathrm{n}+1}=\mathrm{E}_{0, \mathrm{n}-1}^{\infty}
\end{aligned}
$$

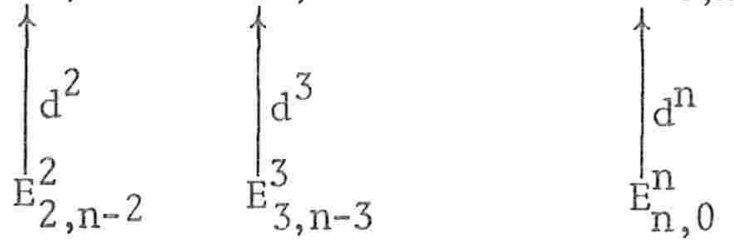

Pela hipótese de indução, sabemos que $H_{j} X=E_{j, n-j}^{2}=0$, $2 \leq j \leq n-1$. Portanto, para os mesmos valores de $j, E_{j, n-j}^{j}=0$. Isso mostra que

$$
E_{0, n-1}^{2}=E_{0, n-1}^{n}
$$

Por outro lado, usando-se o fato que que

$$
\begin{gathered}
E_{0, n-1}^{n+1}=E_{0, n-1}^{\infty}=0, E_{n, 0}^{n+1}=E_{n, 0}^{\infty}=0 \\
\text { (o espaço EX è contractil:) }
\end{gathered}
$$


e

$$
E_{-n,-2 n-2}^{n}=0, E_{2 n,-n+1}^{n}=0
$$

conclui-se facilmente que

$$
E_{0, n-1}^{n}=E_{n, 0}^{n}
$$

Atravës de (1) e (2), obtemos

$$
H_{n-1} \Omega X=E_{n, 0}^{n}
$$

Consideremos agora este outro diagrama tambëm extraido da sequêencia espectral:

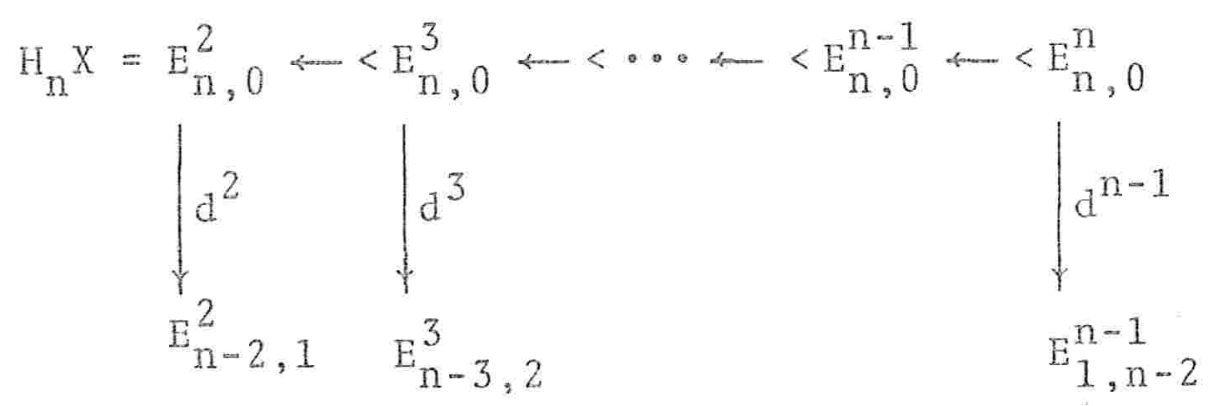

Utilizando-se novamente a hipötese de indução, temos

$$
H_{n-j}\left(X, H_{j-1} \Omega X\right)=E_{n-j, j-1}^{2}=0, \quad 2 \leq j \leq n-1
$$

- que nos mostra que

$$
E_{n-2,1}^{2}=E_{n-3,2}^{3}=\ldots=E_{1, n-2}^{n-1}=0
$$

e portanto que

$$
H_{n} X=E_{n, 0}^{n}
$$


De (3) e (4), concluỉmos que $\mathrm{H}_{\mathrm{n}-1} \Omega \mathrm{X} \longrightarrow \mathrm{H}_{\mathrm{n}} \mathrm{X}$ ê um isomorfismo, o que demonstra o passo de indução do teorema.

Para provar o teorema no caso $\mathrm{n}=2$, basta observar que, como $\pi_{1} \Omega \mathrm{X}$ é abeliano, o homomorfismo $\pi_{1} \Omega \mathrm{X} \rightarrow \mathrm{H}_{1} \Omega \mathrm{X}$ que figu ra no diagrama

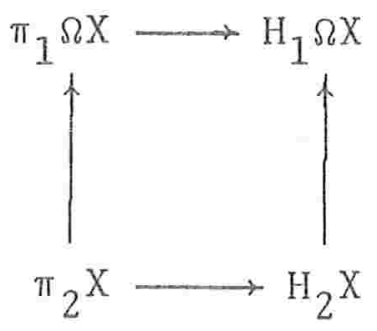

ê um isomorfismo. 圆

Esse teorema pode ser relativizado da seguinte forma:

1.1.2 TEOREMA de Hurewicz relativo. Seja $(X, A)$ um par de es paços tal que $A$ e $X$ são conexos e $\pi_{1} A=\pi_{1}(X, A)=0$. São $\underline{e}$ quivalentes as condições:

i) $\pi_{j}(X, A)=0, \quad 1 \leq j \leq n-1$

ii) $H_{j}(X, A)=0 \quad 1 \leq j \leq n-1$

No caso de uma dessas condições estar satisfeita, temos que

iii) $\pi_{n}(X, A) \longrightarrow H_{n}(X, A)$ é um isomorfismo.

E possive1 demonstrar-se diretamente esse teorema, sem fa zermos apelo ao caso absoluto.

$\mathrm{Na}$ realidade, a demonstração prova ambos os casos simul- 
taneamente. A exemplo do caso absoluto, ê feita por indução sobre o inteiro $n$.

DEMONSTRAÇÃo (esboço de) do teorema 1.1 .2$.

Provaremos que i) $\Rightarrow$ ii) e iii)

início da indução $(n=2)$.

Como $\pi_{1} A=\pi_{1}(X, A)=0$, temos tambëm que $\pi_{1}=0$.

Portanto, no diagrama comutativo abaixo

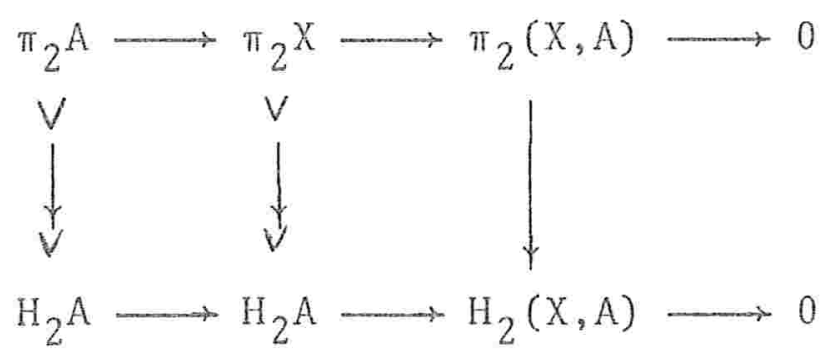

cujas linhas são exatas, as duas flechas verticais à esquer da são isomorfismos (Hurewicz absoluto) e portanto

$$
\pi_{2}(X, A) \rightarrow H_{2}(X, A)
$$

tambëm o è.

Examinemos agora o passo de indução.

Consideremos a fibração

$$
\Omega X \longrightarrow E X, E(X, A) \longrightarrow X, A
$$

onde $\Omega X$ e EX são, respectivamente, os espaços dos laços e, dos caminhos em $X$ e $E(X, A)$ è o espaço dos caminhos em $X$ com extremidade em $A$. 
Valem, para a seqüência espectral associada a essa fibra. ção, as igualdades (ver $\left.[S]_{2}\right)$.

$$
\begin{aligned}
& E_{p, q}^{2}=H_{p}\left(X, A, H_{q} \Omega X\right) \\
& E_{n, 0}^{\infty}=H_{n}(E X, E(X, A))
\end{aligned}
$$

Consideremos o seguinte diagrama extraỉdo da seqüência es pectral

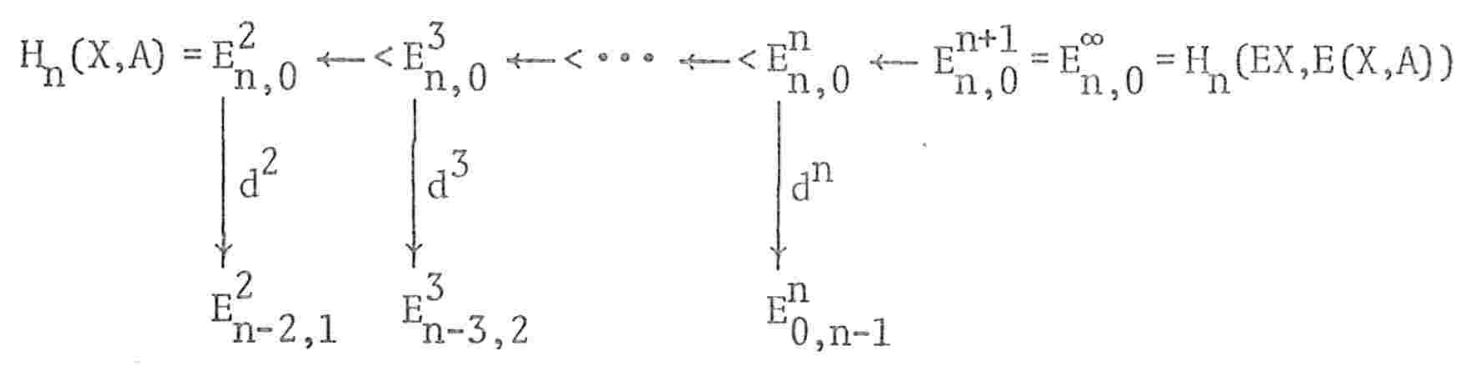

Como

$$
\mathrm{H}_{0}\left(\mathrm{X}, \mathrm{A} ; \mathrm{H}_{\mathrm{n}-1} \Omega \mathrm{X}\right)=0
$$

e, pela hipótese de indução

$$
H_{n-j}\left(X, A ; H_{j-1} \Omega X\right)=0, \quad 1 \leq j \leq n-1
$$

temos que

$$
\mathrm{E}_{n-2,1}^{2}=\mathrm{E}_{n-3,2}^{3}=\ldots=\mathrm{E}_{0, n-1}^{\mathrm{n}}=0
$$

e portanto

$$
\mathrm{H}_{\mathrm{n}}\left(\mathrm{EX}, \mathrm{E}(\mathrm{X}, \mathrm{A}) \rightarrow \mathrm{H}_{\mathrm{n}}(\mathrm{X}, \mathrm{A})\right.
$$

è um isomorfismo. 
Sendo EX conträtil, as sequências exatas longas de homotopia e homologia nos fornecem os isomorfismos

$$
\begin{aligned}
& H_{n}(E X, E(X, A)) \longrightarrow H_{n-1} E(X, A) \\
& \pi_{n}(E X, E(X, A)) \longrightarrow \pi_{n-1} E(X, A)
\end{aligned}
$$

Consideremos então o diagrama comutativo

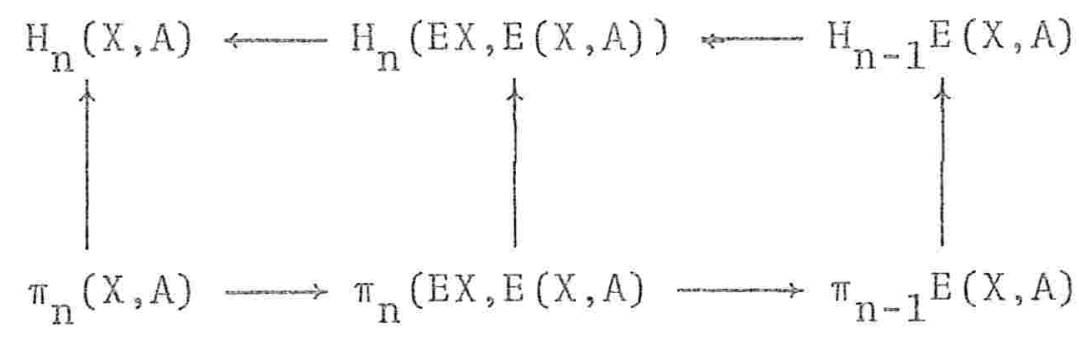

Todas as flechas horizontais são isomorfismos. Para a de monstração de que $\pi_{n}(E X, E(X, A)) \longrightarrow \pi_{n}(X, A)$ e um isomorfismo, o leitor pode consultar $[S]_{2}$, proposição 1 do capítulo II. Como $E(X, A)$ ê n-2 conexo, a hipötese da indução nos garante que a flexa vertical da direita ê um isomorfismo. Em consequência, a flecha vertical da esquerda tambëm o é. Temos, assim, o passo da indução.

o teorema de Hurewicz relativo admite o seguinte 1.1.3 COROLÅRIO. Sejam $X$ e $Y$ espaços 1 -conexos e $X \stackrel{f}{\rightarrow} Y u-$ ma aplicação contínua. Säo equivalentes as condições:

i) $\mathrm{H}_{j} \mathrm{X} \stackrel{\mathrm{E}^{*}}{\longrightarrow} \mathrm{H}_{\mathrm{j}} \mathrm{Y}$ è un isomorfismo para $\mathrm{j}<\mathrm{n}$ e um epimorfis mo para $j \leq n$ 。

ii) $\pi_{j} X \stackrel{f^{*}}{\longrightarrow} \pi_{j} Y$ ë um isomorfismo para $j<n$ e um epimorfis 
mo para $\mathrm{j} \leq \mathrm{n}$.

DEMONSTRAÇAO

Considerando a aplicação cilindro (mapping cylinder) da função $X \stackrel{f}{\longrightarrow} Y$ temos o diagrama comutativo

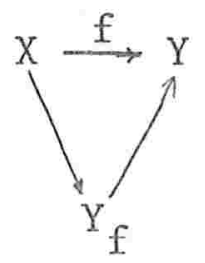

onde $\mathrm{X} \longrightarrow \mathrm{Y}_{f} \overrightarrow{\mathrm{e}}$ uma inclusão e $\mathrm{Y}$ um retrato de deformação de $\mathrm{Y}_{\mathrm{f}}$

Isso mostra que, sem perda de generalidade, podemos considerar a função $X \stackrel{f}{\longrightarrow} Y$ como sendo uma inclusão de $X$ em $Y$.

Utilizando-se, então, as sequências exatas longas de homologia e de homotopia, respectivamente, obtemos as equiva1ências

(i) $\Longleftrightarrow H_{j}(X, Y)=0 \quad j \leq n$

(ii) $\Longleftrightarrow \pi_{j}(X, Y)=0 \quad j \leq n$

onde (i) e (ii) são as condições do enunciado do corolário. Ap1icando-se o teorema 1.1.2, temos a tese.

Esse corolärio, que, em geral, ê falso quando os espaços $X$ e $Y$ não são 1 -conexos (no pröximo capitulo daremos um exem 
plo), adquixe ainda maior substância se lembrarmos que, como nossos espaços são C.W. complexos, vale a

1.1.4 PROPOSIÇÃO. Se a função continua $X \stackrel{f}{\longrightarrow} Y$ induz isomor fismos $\pi_{j} X \stackrel{f^{*}}{\longrightarrow} \pi_{j} Y$ em todas as dimensões, então $f$ ê uma equivalência de homotopia.

As informações contidas no Corolärio 1.1 .3 e na proposição 1.1 .4 são, habitualmente, reunidas num ünico enunciado, conhecido como teorema de Whitehead.

A importância prătica do corolärio 1.1.3, que permite de tectar equivalência de homotopia pelos isomorfismos induzidos na homologia, fez com que os topölogos concentrassem seus esforços nos espaços simplesmente conexos. Todavia, sa be-se hoje que o corolärio 1.1 .3 ê välido para espaços nilpotentes. 
§2. SEQUENCIA EXATA DE J.H.WHITEHEAD

Adendo ao Teorema de Hurewicz

1.2.0. Em 1950, J.H.Whitehead publicou um 1ongo artigo (ver [W $]_{2}$ ) cujo tema central era o estudo de uma sequência exata da qual daremos uma rảpida descriçäo.

Indicaremos por $K$ un C.W. complexo, que suporemos 1-cone xo, e por $K^{p}$ o seu p-esqueleto. $\Gamma_{p} K$ denotará a imagem do ho momorfismo $\pi_{p}\left(K^{p-1}\right) \longrightarrow \pi_{p}\left(K^{p}\right)$ induzido pela inclusão

$$
\mathrm{K}^{\mathrm{p}-1} \longrightarrow \mathrm{K}^{\mathrm{p}} \text {. }
$$

Whitehead mostrou então que, nessas condições, pode-se construir homomorfismos

$$
\begin{gathered}
\mathrm{H}_{\mathrm{p}+1} \mathrm{~K} \stackrel{\gamma_{\mathrm{p}+1}}{\longrightarrow} \Gamma_{\mathrm{p}} \mathrm{K} \\
\Gamma_{\mathrm{p}} \mathrm{K} \stackrel{\lambda_{\mathrm{p}}}{\longrightarrow} \pi_{\mathrm{p}} \mathrm{K},
\end{gathered}
$$

de forma que, se $\pi_{p} K \stackrel{h_{p}}{\longrightarrow} H_{p} K$ è o homomorfismo de Hurewicz, então a sequência

$$
\cdots \longrightarrow \mathrm{H}_{\mathrm{p}+1} \mathrm{~K} \stackrel{\gamma_{\mathrm{p}+1}}{\longrightarrow} \Gamma_{\mathrm{p}} \mathrm{K} \stackrel{\lambda_{\mathrm{p}}}{\longrightarrow} \pi_{\mathrm{p}} \mathrm{K} \stackrel{{ }_{\mathrm{h}}}{\longrightarrow} \mathrm{H}_{\mathrm{p}} \mathrm{K} \longrightarrow \cdots
$$

è exata.

$\mathrm{Na}$ realidade, Whitehead não impöe, a priori, que $\mathrm{K}$ seja simplesmente conexo. A construção ë feita no caso geral e quando $K$ ê simplesmente conexo a sequência é a que descrevemos acima. 
Para as definições do homomorfismos $\gamma_{p}$ e $\lambda_{p}$ " bem como pa ra a prova da exatidão da seqtência, o leitor poderä consuI tar $[H]_{1}$ ou $[M, T]$. Nesta ültima referência, a dedução é feita utilizando-se pares exatos (exact couples), cujas condições de convergência são asseguradas pelo teorema relativo de Hurewicz.

1.2.1. Da exatidão da sequência acima, deduz-se facilmente um adendo ao teorema de Hurewicz. De fato, suponhamos que $K$ $\ddot{e}(N-1)$ conexo. Nessas condições, a inclusão $K^{N-1} \longrightarrow K^{n} \vec{e}$ homotopicamente trivial e, portanto,

$$
\Gamma_{p}(K)=0 \text { para } p \leq n
$$

E claro que isso implica então que

$$
\pi_{n+1} \mathrm{~K} \longrightarrow \mathrm{H}_{\mathrm{n}+1} \mathrm{~K}
$$

$\vec{e}$ um epimorfismo.

E oportuno chamar a atenção do leitor para um fato inte ressante relacionado com a demonstração do resultado acima.

Poder-se-ia pensar em fazer a demonstração do adendo da mesma forma pela qual demonstramos o teorema, isto $\overrightarrow{\mathrm{e}}$, atravës de um argumento de indução, utilizando o diagrama

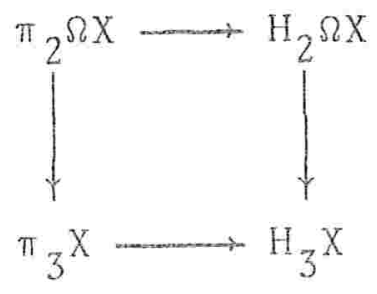


Surge, porêm, um problema, como mostra o seguinte

\subsubsection{EXEMPLO}

Se $X \vec{e}$ simplesmente conexo, jä vimos que $\pi_{3} X \longrightarrow \mathrm{H}_{3} \mathrm{X} \quad \overrightarrow{\mathrm{e}}$ um epimorfimo.

Não $\vec{e}$ verdade, porëm, que $x$ 1-conexo implique que

$$
\pi_{2} \Omega \mathrm{X} \longrightarrow \mathrm{H}_{2} \Omega \mathrm{X}
$$

seja um epimorfismo.

De fato, seja $X=K(\pi, 2)$ e portanto $\Omega X=K(\pi, 1)$.

E öbvio então que $\pi_{2} \Omega X=0$.

Para que se tenha o exemplo, basta que se encontre umes paço $X$ tal que $\mathrm{H}_{2} \Omega \mathrm{X} \neq 0$.

Lembrando que $\mathrm{H}_{2} \Omega \mathrm{X}=\mathrm{H}_{2} \pi$ e, us ando-se resultados elemen tares de (co)homologia de grupos, verifica-se trivialmente que, se fizermos

$$
\pi=\mathrm{Z} \oplus \mathrm{Z}_{2} \text {, temos } \mathrm{H}_{2} \Omega \mathrm{X}=\mathrm{Z}_{2} \text {. }
$$

Complementaremos a observação dizendo que a prova do re sultado em questão pode ser feita por outras vias, sem se re correr à sequência exata de Whitehead. Contudo, uma vez que iríamos, de qualquer modo, introduzir essa sequência exata, pois, no capítulo VI, provaremos uma propriedade dos grupos $\Gamma_{p} \mathrm{~K}$, escolhemos percorrer o caminho acima. 
Existe ainda um outro ponto para o qual queremos chamar a atenção.

A implicação

X 1 -conexo $\Longrightarrow \pi_{3} X \longrightarrow H_{3} X$ è um epimorfismo

permite, atravẻs do mesmo argumento de indução que utilizamos na prova do teorema relativo de Hurewicz, que se demons tre o adendo no caso relativo.

Para referência posterior enunciamos, então, o

1.2.3 TEOREMA de Hurewicz relativo + adendo.

Consideremos o par $(X, A)$ onde $X$ e $A$ são conexos e $\pi_{1} A=$ $=\pi_{1}(X, A)=0$. São equivalentes as condições:

i) $\pi_{j}(X, A)=0 \quad 1 \leq j \leq n-1$

$$
\text { n. } \geq 2
$$

ii) $H_{j}(X, A)=0 \quad 1 \leq j \leq n-1$

$\mathrm{Na}$ eventualidade de uma dessas condiçöes estar satisfei ta, podemos afirmar que

iii) $\pi_{n}(X, \hat{A}) \longrightarrow H_{n}(X, A) \vec{e}$ um isomorfismo

iv) $\pi_{n+1}(X, A) \rightarrow H_{n+1}(X, A)$ è um epimorfismo. 


\section{A TEORIA DE SERRE}

$\S 0$ - INTRODUÇĀO

Em 1952, J.P.Serre, num conhecido trabalho (ver [S] 2 ) in troduziu o conceito de classe de grupos abelianos:

"Une classe c est, par dëfinition, une collection de groupes abêliens que vërifie certaines conditions algêbriques simples. Ces conditions expriment essentieliement que $C$ estestable vis-à-vis des opërations de 1'algébre ëlêmentaire: sous-groupes, groupe quotient, extension. La donnée d'une classe C permet d'introduire des "C-notions" ou 1'on "nëglige" les groupes de la classe C (par exemple, un C-iso morphisme est un homomorphisme dont le noyau appartientà $C$ )"

A utilização dessas classes permitiu-1he, entre outras coisas, uma ampla generalização dos teoremas clássicos de Hurewicz e Whitehead. 
Uma vez que, na parte final deste trabalho, utilizamos e damos uma forma ainda mais geral para alguns desses resul tados, é sensato que se os reproduza aqui.

Como todo esse material $\vec{e}$ hoje clässico e as referencias bibliográficas de que se dispōe para seu estudo muito boas, procederemos, a exemplo do capitulo anterior, de forma bastante esquemätica.

Desenvolvemos, no parägrafo um, a parte da teoria das classes de Serre de grupos abelianos que iremos utilizar. o leitor interessado encontrará, ao lado de um desenvolvimento bem mais amplo da teoria, inumeros exemplos e observaçöes interessantes no artigo acima mencionado.

O segundo e ültimo parägrafo è dedicado às generalizações dos teoremas de Hurewicz e Whitehead. Sem nenhuma pretensão a sermos completos, apenas tentando ilustrar as ideias e os métodos utilizados, enunciamos os resultados de que teremos necessidade e esboçamos algumas demonstraçöes a titulo de exemplo. 
$\S 1$ - CLASSES DE SERRE DE GRUPOS ABELIANOS

Faremos agora um pequeno apanhado das definições e propriedades das classes de Serre de grupos abelianos que iremos utilizar no decorrer do capitulo.

Não nos preocupamos com os aspectos conjuntistas da ques tão, assumindo com relação a eles uma atitude totalmente in gênua. Apesar disso, queremos chamar a atenção do leitor para o fato de que o termo classe (ou coleção) è usado aqui no sentido que the $\vec{e}$ dado na teoria dos conjuntos não sendo por tanto sinônimo de conjunto.

2.1.0. Uma classe de Serre de grupos abelianos ë uma coleção, não vazia, $C$ de grupos abelianos satisfazendo a condição

I) Se $A, A^{\prime}, A^{\prime \prime}$ são grupos abelianos e a sequência

$$
0 \rightarrow A^{\prime} \longrightarrow A \longrightarrow A^{\prime \prime} \longrightarrow 0
$$

$\vec{e}$ exata, então vale a equivalência

$$
A^{\prime}, A^{\prime \prime} E C \Longleftrightarrow A \in C \text {. }
$$

Decorre imediatamente dessa definição que

a) todo grupo com um ünico elemento estä em $C$.

c) todo grupo isomorfo a um subgrupo ou a um grupo quociente de um grupo de $C$ está em $C$.

2.1.1. Frequentemente, para um melhor desenvolvimento da teo 
ria, precisamos impor alguns axiomas suplementares à classe C.

Mencionaremos os que nos serão üteis.

$\left.I_{A}\right) A \in C, B \in C \Longrightarrow A \otimes B$ etor $(A, B) \in C$.

$I_{B}$ ) $A \in C \Longrightarrow A \otimes B \in C$ qualquer que seja o grupo abeliano B。

Prova-se, sem dificuldade que II $_{B}$ implica II $_{A}$.

III) $\mathrm{AEC} \Longrightarrow \mathrm{H}_{\mathbf{i}} \mathrm{AEC}, \mathrm{i} \geq 1$.

Chamaremos de aciclicas as classes verificando esseaxio ma.

A existência ou não de classes não verificando esse axio ma ë, pelo que o autor sabe, um problema aberto.

IV) Qualquer soma direta (finita ou não) de grupos de c estä em C.

As classes que verificam esse axioma serão chamadas de completas (confronte 3.2 .0 ).

Prova-se (ver $[\mathrm{S}]_{2}$ ) que esse axioma implica na validade de $I_{A}, I I_{B}$ e III.

Com frequência utiliza-se classes verificando simultaneamente os axiomas II $_{A}$ e III ou II $I_{B}$ e III. Eis portanto a 1 guns exemplos: 
2.1.3. Classes verificando os axiomas II $\mathrm{A}$ e III

1) A classe trivial, constituida pelos grupos com um $\vec{u}$ nico elemento.

2) A classe dos grupos abelianos finitamente gerados.

3) A classe dos grupos abelianos finitos.

4) A classe dos grupos finitos cuja ordem sỏ ê divis $\overrightarrow{i-}$ vel por uma familia dada de primos.

2.1.4. Classes verificando os axiomas $I_{B}$ e III

1) A classe trivial.

2) A classe dos grupos abelianos de torção.

3) A classe dos grupos abelianos de torção cujas p-componentes são nulas para uma familia dada de primosp.

Esses exemplos verificam tambëm o axioma IV.

A proposição abaixo, cuja prova damos, no capítulo IV (veja lema 4.1.0) nos será de utilidade.

2.1.5. PROPOSIÇÃO - Sejam A e B grupos abelianos com B em C. Se A for finitamente gerado ou se a classe $C$ for completa, então:

i) $B \otimes A \in C$

ii) $\operatorname{Tor}(B, A) \in C$.

2.1.6. As C-noções

Ao considerarmos uma classe de grupos $C$ a idêia geral ẻ podermos, em certo sentido, negligenciar os grupos de $C$, is 
to $\vec{e}$, trat $\vec{a}-10 s$ como grupos triviais.

Formalizamos essa idëia, com as definiçöes abaixo.

Diremos que um homomorfismo $A \stackrel{\alpha}{\longrightarrow} B$ de grupos abelianos é C-injetor se ker aEC e, C-sobrejetor se coker aEC. Quando o homomorfismo for $C$-injetor e C-sobrejetor diremos que ele è c-bijetor.

Nesse ponto $\vec{e}$ instrutivo darmos um exemplo.

Seja C a classe dos grupos abelianos de torçäo, $\mathbb{Z}$ o gru po dos inteiros e Q o grupo dos racionais.

Uma vez que $Q / \mathbb{Z}$ e um grupo de torção, a inclusão $\mathbb{Z} \underset{\rightarrow}{\rightarrow}$ $\vec{e}$, obviamente, uma c-bijeção.

Prova-se, no entanto, trivialmente que o ünico homomorfismo de $Q$ em $\mathbb{Z}$ èo homomorfismo nulo que, obviamente, não $\vec{e}$ uma C-bijeção.

Contorna-se essa dificuldade com uma definição.

Dois grupos $A \in B$ são $C$-isomorfos se existe um grupo $L$ e dois homomorfismos, $L \stackrel{f}{\longrightarrow} A$ e $L \stackrel{g}{\rightarrow} B$, ambos C-bijetores.

Pode-se provar que essa relação $\bar{e}$ transitiva.

Terminamos observando que valem para $C$-bijeções. e $C$-sobrejeções as mesmas propriedades das injeçöes e sobrejeçōes usuais.

Pode-se, por exemplo, provar facilmente que se 


$$
\begin{gathered}
-25- \\
\mathrm{E} \stackrel{\mathrm{I}}{\longrightarrow} \mathrm{F} \stackrel{\mathrm{g}}{\rightarrow} \mathrm{G}
\end{gathered}
$$

são homomorfismos de grupos abelianos então valem:
a) $f, g C$-injeções $\Longrightarrow g \circ f($-injeções
b) $\mathrm{f}, \mathrm{g} \mathrm{C}$-sobrejetores $\Longrightarrow$ gf $\mathrm{C}$-sobrejetor
c) $g \circ f(-$-injetor $\Rightarrow f(c$-injetor. etc. 
$\S 2$ - TEOREMAS DE HUREWICZ E DE WHITEHEAD MỎDULO C

Veremos agora de que forma as classes de Serre de grupos abelianos permitem generalizar os resultados do capi tulo anterior.

Em todo este parägrafo, $c$ indicarä uma classe de Serre aciclica de grupos abelianos. Quando precisarmos que a classe verifique a algum outro axioma, mencionaremos o fa to explicitamente.

Começaremos examinando o caso absoluto.

2.2.0 TEOREMA. Seja $C$ uma classe verificando o axioma II $_{A} e$ $X$ um espaço conexo e simplesmente conexo. Säo equivalentes
i) $\pi_{j} \mathrm{XEC}$
$1 \leq j \leq n-1$
i i) $\mathrm{H}_{\mathrm{j}} \mathrm{XEC}$
$1 \leq j \leq n-1$.

No caso de uma dessas condições estax verificada, podemos afirmar que:

iii) $\pi_{n} \times \longrightarrow H_{n} \times \vec{e}$ uma C-bijeção

iv) $\pi_{n+1} X \longrightarrow H_{n+1} X$ è uma c-sobrejeção.

Daremos un esquema da demonstração de que i) $\Longrightarrow$ ii)

e iij). Como de häbito, faremos indução sobre n.

Como jầ provamos que se $\pi_{1} X=0$ então $\pi_{2} X \rightarrow \mathrm{H}_{2} \mathrm{X}$ é un isomorfismo, a indução principia trivialmente. 
Para provarmos o passo da indução $(n \geq 3)$ introduzi mos a fibração

$$
\Omega \mathrm{X} \longrightarrow \mathrm{EX} \longrightarrow \mathrm{X}
$$

e o diagrama comutativo

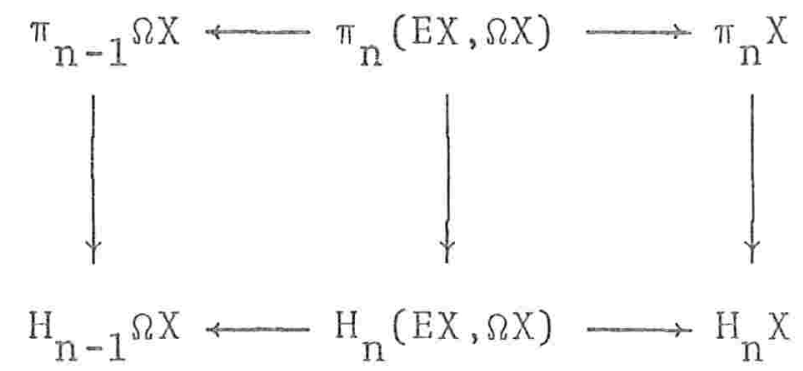

onde as flechas verticais são os homomorfismos de Hurewicz, $\pi_{n}(E X, \Omega X) \longrightarrow \pi_{n} X \vec{e}$ o isomorfismo que se utiliza para deduzir a sequência exata de uma fibração,

$$
\begin{aligned}
& \pi_{n}(E X, \Omega X) \longrightarrow \pi_{n-1} \Omega X \\
& H_{n}(E X, \Omega X) \longrightarrow H_{n-1} \Omega X
\end{aligned}
$$

são isomorfismos deduzidos, respectivamente, das sequências exatas longas de homotopia e de homologia e,

$$
\mathrm{H}_{\mathrm{n}}(\mathrm{EX}, \Omega X) \longrightarrow \mathrm{H}_{\mathrm{n}} \mathrm{X}
$$

$\vec{e}$ um homomorfismo que se prova, usando a hipótese de indução e a sequência espectral associada a um fibrado relativo, ser um isomorfismo (confronte $[S]_{2}$, Teorema 1.A).

Se provarmos que $\pi_{n-1} \Omega \mathrm{X} \longrightarrow \mathrm{H}_{n-1} \Omega \mathrm{X}$ è uma $c-b i j e c ̧ a ̃ o$, 
teremos que $\pi_{n} X \longrightarrow{ }_{n} X$ também o $\vec{e} e$, portanto, estarä esta belecido o passo da indução.

Não podemos concluir diretamente da hipötese de in-

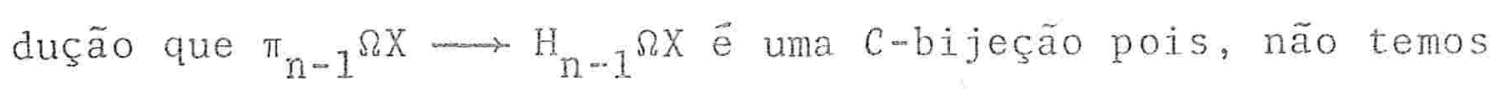
garantia de que $\Omega X \vec{e} I$-conexo.

Seja então $\mathrm{T} \longrightarrow \Omega \mathrm{X}$ o recobrimento universal de $\Omega \mathrm{X}$ e, consideremos o diagrama comutativo

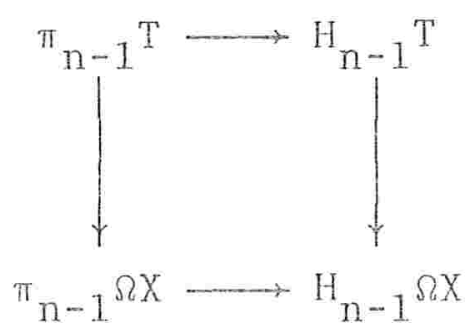

onde a flecha vertical da esquerda $\vec{e}$, obviamente, um isomorfismo.

Como $\mathrm{T} \overrightarrow{\mathrm{e}} I$-conexo e $\pi_{j} \mathrm{TEC}, 1 \leq j \leq n-2$, a hipótese de indução, nos fornece que

$$
\pi_{n-1}{ }^{\mathrm{T}} \longrightarrow \mathrm{H}_{n-1} \mathrm{~T} \text { è uma } \mathrm{C}-\text { bijeção. }
$$

Basta provar então que $\mathrm{H}_{\mathrm{n}-1} \mathrm{~T} \longrightarrow \mathrm{H}_{\mathrm{n}-1} \Omega \mathrm{X}$ è uma $\mathrm{C}$-bi jeção e teremos que

$$
\pi_{n-1} \Omega X \longrightarrow H_{n-1} \Omega X
$$

tambêm o será.

A prova de que ${ }_{n-1}^{T} \longrightarrow H_{n-1}{ }^{T}$ ë uma C-bijeção e feita utilizando-se a sequência espectral do recobrimento $\mathrm{T} \longrightarrow \Omega \mathrm{X}$ (veja o parägrafo dois do capitulo V), e envolve um 
argumento anâlogo ao que usamos para provar a implicação i) $\Longrightarrow$ ii) do teorema 5.3 .4 do capítulo V. Aqui, como $\pi_{1} \Omega X$ atua trivialmente em $\mathrm{H}_{\mathrm{j}}^{\mathrm{T}}$ (ver $[\mathrm{S}]_{1}$, päg. 479), a verificação de que os conücleos relevantes estão em $C$ ê feita usando-se a sequência exata

$$
0 \longrightarrow \mathrm{H}_{\mathrm{p}} \pi \otimes \mathrm{H}_{\mathrm{q}} \mathrm{T} \longrightarrow \mathrm{H}_{\mathrm{p}}\left(\pi, \mathrm{H}_{\mathrm{q}} \mathrm{T}\right) \longrightarrow \mathrm{Tor}\left(\mathrm{H}_{\mathrm{p}-1} \pi, \mathrm{H}_{\mathrm{q}} \mathrm{T}\right) \longrightarrow 0
$$

onde $\pi=\pi_{I} \Omega X$

fornecida pelo teorema dos coeficientes universais e lembran do que a classe $C$ verifica o axioma II $_{A}$.

Não è difïcil ver que o teorema acima, em geral, não é verdadeiro se $\pi_{1} X \neq 0$. No capitulo $\mathrm{V}$ fornecemos um exemplo desse tipo.

No entanto, se o espaço $x$ for tal que $\pi_{1} x \vec{e}$ abelia no, pertence a $C$ e opera trivialmente nos grupos de homologia de seu revestimento o teorema acima è verdadeiro para $\mathrm{X}$, (conforme observação 2 da pägina 274 de $[S]_{2}$ ).

Esse fato è utilizado na demonstração do seguinte 2.2.1. TEOREMA. Seja $C$ uma classe verificando o axioma $I_{B}$ * Sejam X e A dois espaços conexos e 1-conexos com AcX. São e quivalentes as condições:
i) $\pi_{j}(X, A) \in C$
$1 \leq \mathrm{j} \leq \mathrm{n}-1$
ii) $\mathrm{H}_{j}(X, A) \in C$
$1 \leq \mathrm{j} \leq \mathrm{n}-1$. 
no caso de uma dessas condições estar verificada, podemos a firmar que

$$
\begin{aligned}
& \text { iii) } \pi_{n}(X, A) \longrightarrow H_{n}(X, A) \text { è uma c-bijeção } \\
& \text { iv }) \pi_{n+1}(X, A) \longrightarrow H_{n+1}(X, A) \vec{e} \text { uma c-sobrejeção. }
\end{aligned}
$$

Para a demonstração, que è o anälogo na $\mathrm{C}$-teoriada demonstração do teorema 1.12 , ver $[S]_{2}$.

Chamamos a atençäo do leitor para o fato de que a imposição, no enunciado dos teoremas acima, de que a classe $C$ verifique os axiomas $I_{A}$ num caso $I_{B}$ no outro se deve à necessidade de nos assegurarmos de que se $A \in C$ (e, no casoda classe $C$ verificar somente $\left.I_{A}, B \in C\right)$ então $A \otimes B \quad C \operatorname{Tor}(A, B)$ tambêm estão em $C$.

Levando-se então em conta que a proposição 2.1.5 nos garante que se $A \vec{e}$ grupo de $C$ então $A \otimes B$ e $\operatorname{Tor}(A, B)$ estão em $C$ desde que $C$ seja completa ou B finitamente gerado, vemos que os teoremas 2.2 .0 e 2.2 .1 permanecem verdadeiros se trocarmos a exigência de que a classe C verifique os axiomas II A II $_{B}$ pela condição dos espaços envolvidos serem de tipo finito ou então que a classe C seja completa.

o teorema 2.2 .1 admite, como corolärio, o seguinte 2.2.2 TEOREMA. Suponhamos que $C$ verifique o axioma $I_{B}$. Sejam $A$ e $X$ espaços conexos 1 -conexos e $A \stackrel{f}{\longrightarrow} X$ uma aplicaçäo continua. São equivalentes as condições: 
i) $H_{j}(A) \stackrel{f^{*}}{\longrightarrow} H_{j} X$ è uma $C$-bijeção para $1 \leq j<n$ e uma $C$ sobrejeçäo para $j=n$.

ii) $\pi_{j}(A) \stackrel{f^{*}}{\longrightarrow} \pi_{j} X \vec{e}$ uma $C$-bijeção para $1 \leq j<n$ e uma $C$ sobrejeção para $\mathrm{j}=\mathrm{n}$.

DEMONSTRAÇÃO

E anāloga a demonstração do corolärio 1.1 .3 (ver $\left.[\mathrm{S}]_{2}\right)$.

Quando a hipötese de que os espaços envolvidos são simplesmente conexos não está verificada, o teorema acima po de deixar de ser verdadeiro como mostra o seguinte

2.2 .3 EXEMPLO

Consideremos a inclusão

$$
S^{1} \longrightarrow S^{1} v S^{2} U_{f} e^{3}
$$

A função $f S^{2} \longrightarrow S^{1} v S^{2}$ usada para a colagem de $e^{3}$ $\vec{e}$ um representante do elemento $2[\alpha]-[\omega] \cdot\left[\alpha_{0}\right] \in \pi_{2}\left(S^{1} v S^{2}\right)$ onde $[\alpha] \vec{e}$ a imagem de um gerador de $\pi_{2}\left(S^{2}\right)$ pela inclusão

$$
S^{2} \longrightarrow s^{1} v S^{2}
$$

e $[\omega] \vec{e}$ a imagem de um gerador de $\pi_{1}\left(S^{1}\right)$ pela inclusão

$$
S^{1} \rightarrow S^{1} \vee S^{2}
$$

$[\omega] \cdot[\alpha] \vec{e}$ o elemento obtido pela ação de $[\omega]$ em $[\alpha]$.

Não è difïcil provar (confronte [Sp], pảgina 420, 
exercício F4) que a inclusão

$$
S^{1} \longrightarrow S^{1} v S^{2} U_{f} e^{3}
$$

induz isomorfismos entre os grupos fundamentais e entre todos os grupos de homologia mas não entre os grupos de homotopia em dimensão dois. 


\section{GRUPOS E AÇÕES NILPOTENTES. CLASSES DE SERRE DE GRUPOS NILPOTENTES. ESPAÇOS NILPOTENTES}

§0. INTRODUÇĀO

Este ë um capítulo de pré-requisitos. Nele o leitor encontrarả definições e enunciados mas nenhuma demonstração.

Não foi nossa intenção fazer um resumo geral dos töpicos que figuram no título mas sim, tão somente, reunir, para facilidade do leitor, num ünico local, as definições e resulta dos que nos pareceram mais especializados e que teremos opor tunidade de utilizar nos capítulos subsequentes.

Iniciamos recordando, apenas com o intuito de fixar a lin guagem, umas poucas noçöes da teoria geral dos grupos. A seguir, definimos açöes nilpotentes e mencionamos algumas de suas propriedades. As melhores referências para esse töpico são $[H]_{1},[H]_{2},[H]_{3}$ e $[H, M, R]$. 
No parägrafo 2 fazemos um apanhado geral da teoria das classes de Serre de grupos nilpotentes. Os resultados a 1 reu nidos são de importância fundamental para o desenvolvimento dos capitulos posteriores. Indicamos como referências [H,M,R] e $[H, R]_{1}$.

Abrimos o îtimo parágrafo com uma pequena propaganda dos espaços nilpotentes. Uma consulta a $[H, M, R]$ ou a [Su] poderá ser, nesse sentido, proveitosa. Ao lado de algumas definições e propriedades gerais enunciamos alguns resultados de cunho bem mais tëcnico. Devemos, com relação a isso, una explicação. Tentando manter sob controle, paxa näo romper o equilíbrio do trabalho, a extensäo do capitulo, acabamos por pressupor do lejtor, neste ponto, e, consequentemente na demonstração do lema 5.3 .3 do capítulo $\mathrm{V}$, um conhecimento especializado um pouco maior do que o pressuposto no resto do traba 1ho. Acreditamos, no entanto, que uma vista d'olhos em $\left[\mathrm{H}_{5}\right.$ dissiparä, caso existam, as dificuldades. As principais refe rências são $[H]_{5},[H, M, R],[H, R]_{1}$ e $[H, R]_{2}$ e, para o leitor interessado em fatos gerais, como, por exemplo, as definiçöes das diversas ações de grupos nas fibrações, [B]. 


\section{\$1. GRUPOS E AÇOES NILPOTENTES}

Começamos lembrando algumas definições.

3.1.0. A sërie central inferior de un grupo $G$ é definida indutivamente pelas igualdades

$$
\Gamma^{1}{ }_{G}=G, \Gamma^{i+1} G=\left[\Gamma^{i} G, G\right] \text {, }
$$

onde $\left[\Gamma^{i} G, G\right]$ indica o grupo dos comutadores de $\Gamma^{i} G$ e $G$.

Convëm observar, no que se refere à definição acima, que a convenção $\Gamma^{0} \mathrm{G}=\mathrm{G}$ è tambëm frequente na 1iteratura.

Quando existir um inteiro positivo $c$ tal que

$$
\Gamma^{\mathrm{C}} \mathrm{G} \neq\{1\} \text { e } \Gamma^{\mathrm{C}+1} \mathrm{G}=\{1\}
$$

diremos que o grupo $G$ ë nilpotente e tem classe de nilpotência c.

Segue-se imediatamente dessa definição que todo grupo abeliano $\vec{e}$ nilpotente. $\vec{E}$, por outro lado, bem conhecido que nem todo grupo nilpotente ë abeliano. Demonstra-se, por exem plo, que todo p-grupo finito ë nilpotente.

3.1.1. Seja G um grupo e H um subgrupo de G consideremos uma sërie de subgrupos

$$
H=G_{0} \subset G_{1} \subset \ldots c G_{k} \subset \ldots c G_{n}=G
$$

Iigando $\mathrm{H}$ a $\mathrm{G}$. 
Diremos que essa sêrie è normal se

$$
\mathrm{G}_{\mathrm{k}} \triangleleft \mathrm{G} \quad \mathrm{k}=0, \ldots, \mathrm{n}-1
$$

A notação $G_{k} \triangleleft G$ indica, como de häbito, que $G_{k} \vec{e}$ um subgrupo normal de G.

Se tivermos apenas que

$$
\mathrm{G}_{\mathrm{k}} \triangleleft \mathrm{G}_{\mathrm{k}+1} \quad \mathrm{k}=0, \ldots, n-1
$$

a sërie $\ddot{e}$ chamada subnormal.

Um subgrupo $H$ do grupo $G \vec{e}$, por definiçäo, um subgrupo subnormal de G se existir uma sêrie subnomal

$$
H=G_{0} \subset G_{1} \subset \ldots c G_{n}=G
$$

Iigando $\mathrm{H}$ a $\mathrm{G}$.

A noçäo de subgrupo subnormal generaliza a de subgrupo normal pois ê öbvio que um subgrupo normal ë tambëm subnorma1. A reciproca porẻm não ë verdadeira. Tambëm näo è, en ge ral, verdade que todo subgrupo ê subnormal.

Vale porëm o seguinte resultado, que nos serả de grande utilidade.

3.1.2. PROPOSTCAOO. Se $G$ è um grupo nilpotente e $H$ è um subgrupo qualquer de $G$ entäo $H$ e un subgrupo subnormal de G.

o Ieitor encontrarä uma demonstração dessa proposiçãobem como exemplos e demonstraçöes para as afirmaçöes que fizenos 
acima em [R]。

3.1.3. A noção de grupo nilpotente generaliza-se atravës do conceito de ação nilpotente.

Sejam $\pi$ e $G$ dois grupos e suponhamos que $G$ é um $\pi$-grupo, isto ê, que esteja definida uma ação $\pi \stackrel{\omega}{\longrightarrow}$ Aut(G) de $\pi$ em $G$, (simplificaremos a notação escrevendo $x \cdot a$ em vez de $\omega(x) \cdot a)$.

A ação $\omega$ permite-nos construir indutivamente a série $\Gamma_{\omega}^{1} G=G, \quad \Gamma_{\omega}^{i+1} G=g p\left(x \cdot a b a^{-1} b^{-1}\right), x \in \pi, a \in \Gamma{ }_{\omega}^{i} G, b \in G i \geq 1$

onde $g p\left(x \cdot a b a^{-1} b^{-1}\right)$ indica o grupo gerado pelos elementos $(x \cdot a) b^{-1} b^{-1}$.

Se $\Gamma_{\omega}^{c+1} G=\{1\}$ para algum inteiro $c$, diremos que $\pi$ opera nilpotentemente em $G$ e escrevemos classe $(\omega) \leq c$. Se alem dis so $\Gamma_{\omega}^{\mathrm{C}} \mathrm{G} \neq\{1\}$, então classe $(\omega)=c$.

Decorre dessa definição que a ação trivial é nilpotente se e somente se o grupo G ë nilpotente.

E oportuno observar que, embora a nossa definição de ação nilpotente não seja equivalente a dada em $[B, K]$, no caso do grupo G ser nilpotente elas coincidem.

Teremos, no capitulo $\mathrm{V}$, oportunidade de usar duas proprie dades das ações nilpotentes que decorrem, sem dificuldade, da definição: 
3.1.4. Seja $\mathrm{H}$ um subgrupo de $\pi$ e $\pi \stackrel{\omega}{\longrightarrow}$ Aut $(G)$ uma ação nilpo tente. A restrição de w a $\mathrm{H}$ è tambêm uma ação nilpotente.

3.1.5. Se $\pi \stackrel{\omega}{\longrightarrow}$ Aut $(G)$ è uma ação nilpotente e $H \stackrel{\varepsilon}{\longrightarrow}>\pi$ um epimorfismo de grupos então a ação $H \stackrel{\omega^{*} \varepsilon}{\longrightarrow}$ Aut $(G)$, obtida pe la composição de $\omega$ e $\varepsilon$, ë tambëm nilpotente.

$\mathrm{Na}$ demonstração do Iema 5.3 .3 utilizaremos uma outra pro priedade, esta de demonstração mais elaborada, das ações nil potentes.

3.1.6. TEOREMA. Uma ação $\omega$ de $\pi$ em $G$ induz uma ação de $\pi$ nos grupos de homologia $H_{n} G$ de $G$. Se a ação w for nilpotente então a ação induzida nos grupos de homologia tambëm o serä.

Este teorema $\vec{e}$ o 1 ema 1.2 de $[\mathrm{H}] 5^{\circ}$

\subsubsection{OBSERVACAO}

Queremos chamar a atenção do leitor para o fato de que, na maioria das vezes, (a prova do lema 5.3 .3 constitui-se na principal exceção:) estaremos considerando ações de um grupo $\pi$ num grupo abeliano A. Nesse caso, como vantagem adicional, temos o fato de A admitir uma estrutura de Z[T] módulo. Isso e o que acontece, por exemplo, no capítulo IV onde $\pi$ sempre. age sobre um grupo abeliano. E claro que, nessas circunstancias, usando-se a notação aditiva, temos a igualdade

$$
\Gamma_{\omega}^{i} A=g p(x a-a), \quad x \in \pi, \quad a \in \Gamma{ }_{\omega}^{i-1} A, \quad i>1
$$


da qual decorre que

$$
\Gamma_{\omega}^{i+1} A=I[\pi]_{0}^{i} A
$$

onde $I[\pi]$ representa 0 ideal de aumentação do anel de grupo $Z[\pi] .(\operatorname{ver}[H, M, R])$. 
§. CLASSES DE SERRE DE GRUPOS NILPOTENTES

Resumiremos aqui a parte que nos interessa mais de perto da teoria das classes de grupos nilpotentes.

A generalizaçäo para grupos nilpotentes da teoria desenvolvida por Serre no caso abeliano foi feita por Hilton, Mis Iin e Roitberg (ver $[H, M, R]$ e $[H, R])$. Da mesma forma que no caso abeliano, a motivaçäo para essa teoria algëbrica surgiu da Topologia. Mais precisamente do desejo de desenvolver uma teoria de Homotopia para espaços nilpotentes nos moldes do que Serre havia feito no caso simplesmente conexo.

Vejamos agora algumas definições.

3.2.0. DEFINTGAO. Uma classe de Serre de grupos nilpotentes ë uma coleção não vazia $C$ de grupos nilpotentes tal que para qualquer extensão central

$$
\mathrm{N}>\mathrm{G} \longrightarrow \mathrm{G}
$$

de grupos nilpotentes, vale a equivalência

$$
\mathrm{N}, \mathrm{HEC} \Longleftrightarrow \mathrm{GEC}
$$

Diremos que a classe $c$ è pröpria se uma das condições abaixo se verifica

I) Se A è um grupo abeliano de $C$ então $A$ ë finitamente gerado. 
II) Se A è um grupo abeliano de $C$ então $\oplus A \in C$, onde a soma direta inclui um nümero arbitráxio de cópias de A.

Quando a condição II) está satisfeita dizemos que aclas se $\vec{e}$ completa.

Chamaremos de aciclica uma classe $C$ que satisfizer acon dição

$$
\mathrm{G} \in \mathrm{C} \Longrightarrow \mathrm{H}_{\mathrm{n}} \mathrm{G \in C}, \quad \mathrm{n} \geq 1
$$

Na realidade, a exigência de que a condição acima esteja satisfeita apenas pelos grupos abelianos de $c$ ja $\vec{e}$ suficiente para assegurar a aciclicidade da classe C (ver propo sição 5.4 de $[H, M, R])$.

Eis alguns exemplos de classes de Serre de grupos nilpo tentes

1) A classe constituỉda pelos grupos contendo um único elemento. Essa classe é chamada de trivial ou então de classe zero.

2) A classe de todos os grupos nilpotentes.

3) A classe dos grupos nilpotentes finitamente gerados.

4) A classe dos grupos nilpotentes de torção.

5) A classe dos grupos nilpotentes finitos.

6) A classe dos grupos nilpotentes de P-torção onde $\mathrm{p} \overrightarrow{\mathrm{e}} \underline{u}$ ma família de primos.

Dois resultados importantes e que utilizaremos reitera- 
das vezes são dados pelas proposições abaixo. As demonstrações estão em $[H, M, R]$.

3.2. I PROPOSICÃO. Seja $C$ una classe de Serre própria de gru pos nilpotentes. Então GCC se e somente se $G_{a b} \in C$. $G_{a b}$ indica $O$ abelianizado de $G$.

3.2.2 PROPOSICAO . Seja $C$ uma classe de Serre propria de gru pos nilpotentes. Dada uma sequência exata curta de grupos nilpotentes

$$
\mathrm{G}^{\prime}>\mathrm{G} \longrightarrow \mathrm{G}^{\prime \prime}
$$

Vale a equivalência

$$
G^{\prime}, G^{\prime \prime} \in C \Longleftrightarrow G \in C \text {. }
$$

3.2.3. Generalizemos agora para o caso nilpotente, os conceitos de $c$-injeção e c-sobrejeção que introduzimos no capi tulo anterior quando estudamos classes de grupos abelianos. Nas considerações seguintes estaremos supondo que a classe C $\ddot{e}$ pröpria.

E claro que a noção de C-injetividade se adapta sem modificação ao caso nilpotente. Portanto, tambëm neste caso, diremos que o homomorfismo $A \stackrel{\mu_{-}}{\longrightarrow}$ B é uma $C$-injeção quando keruec.

A definição de C-sobrejeção $\vec{e}$, no entanto, em consequên cia do fato da imagem de um homomorfismo não ser necessaria 
mente um subgrupo normal, mais delicada. Contornaremos essa dificuldade explorando o fato de que todo subgrupo de um gru po nilpotente $\vec{e}$ subnormal.

3.2.4 DEFINICAO Seja G um grupo nilpotente e H um subgrupo de G. Diremos que a inclusão de $H$ em $G \vec{e}$ uma $C$-sobrejeção se existir uma sërie subnormal

$$
\mathrm{H}=\mathrm{H}_{0} \triangleleft \mathrm{H}_{1} \triangleleft \ldots \triangleleft \mathrm{H}_{\mathrm{k}+1}=\mathrm{G}
$$

tal que $\mathrm{H}_{\mathrm{j} / \mathrm{H}} \in \mathrm{G}-\mathrm{C}, \mathrm{j}=1, \ldots, \mathrm{k}+1$.

Isto posto, passemos à definição geral.

3.2.5 DEFINIÇÃO. Seja $H \stackrel{\mu}{\longrightarrow}$ G um homomorfismo de grupos niI potentes. O homomorfismo $\mu \vec{e}$ C-sobrejetor se a inclusão de $\mu(H)$ em G for uma C-sobrejeção.

Diremos que $\mu$ è $C$-bijetor se $\mu$ for $C$-injetor e C-sobrejetor.

O leitor encontrarâ uma justificação para essas definições bem como para as propriedades dela decorrentes que figuram na proposição abaixo em $[H, R]_{1}$.

3.2.5 PROPOSICÃO. Consideremos os homomorfismos

$$
\mathrm{H} \stackrel{\lambda}{\longrightarrow} \mathrm{G} \stackrel{\mu}{\longrightarrow} \mathrm{K}
$$

de grupos nilpotentes. Valem as implicações:

i) $\lambda, \mu c$-injetivas $\Longrightarrow \mu \cdot \lambda C$-injetiva. 
ii) $\lambda, \mu c$-sobrejetivas $\Longrightarrow \mu \cdot \lambda c$-sobrejetiva.

iii) $\mu . \lambda C$-injetiva $\Rightarrow \lambda C$-injetiva.

iv) $\mu \cdot \lambda C$-sobrejetiva $\Longrightarrow \mu C$-sobrejetiva.

v) $\mu . \lambda c$-injetiva, $\lambda c$-sobrejetiva $\Longrightarrow \mu c$-injetiva.

vi) $\mu . \lambda c$-sobrejetiva, $\mu c$-injetiva $\Longrightarrow \lambda c$-sobrejetiva.

Um outro resultado - este central na teoria - que iremos utilizar $\hat{\mathrm{e}}$ o seguinte

3.2.6 TEOREMA. (Lema dos cinco módulo c). Seja c uma classe de Serre propria de grupos nilpotentes. Consideremos o diagrama

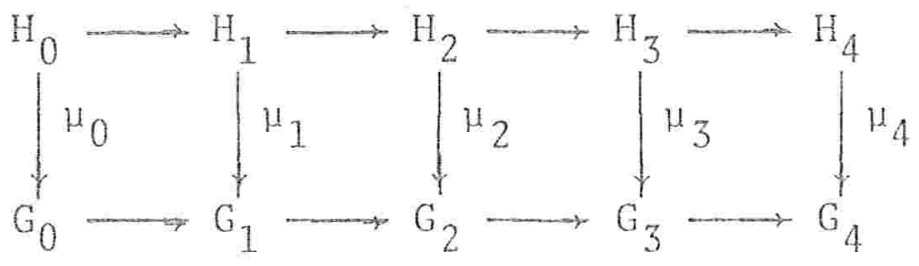

cujas linhas são exatas. Se $\mu_{0}$ è C-sobrejetiva, $\mu_{1}$ e $\mu_{3}$ são C-bijetivas e $\mu_{4} \vec{e}$ C-injetiva então $\mu_{2} \vec{e}$ C-bijetiva.

Reuniremos agora, para referència posterior,algumas pro priedades muito úteis das classes de Serre generalizadas. Suas demonstrações não apresentam nenhuma dificuldades e carão aos cuidados do leitor.

3.2.7. Se GGC e H ê um subgrupo de G então HeG. Utiliza-se, na demonstração, a proposição 3.2 .2 e o fato de todo sub-. grupo de um grupo nilpotente ser subnormal.

$3.2 .8 . B, A / B \in C \Rightarrow A \in C$.

3.2.9. Se $A \stackrel{\mu}{\longrightarrow} B$ è um homomorfismo de grupos e AGC então 
$\operatorname{Im} \mu \in C$ 。

Em particular, se $\mu \overrightarrow{\mathrm{e}}$ um epimorfismo, BEC.

3.2.10. Se a sequência $A^{\prime} \longrightarrow A \longrightarrow A^{\prime \prime}$ é exata e $A^{\prime}, A^{\prime \prime} \in C$ en tão AEC.

Concluiremos com a

\subsubsection{OBSERVAÇAO}

Consideremos uma partição do conjunto dos nümeros primos em duas familias $\mathrm{P}$ e Q. Indicaremos por $C_{Q}$ a classe de Serre dos grupos nilpotentes de Q-torção.

Define-se na teoria de localização dos grupos nilpotentes os conceitos de P-injeção e de P-sobrejeção (confronte $[\mathrm{H}, \mathrm{M}, \mathrm{R}]$ où $\left.[\mathrm{H}]_{2}\right)$.

Verifica-se, sem dificuldade, que esses conceitos coincidem com as noções de $C_{Q}$-injeção e $C_{Q}$-sobrejeção como as de finimos acima. Essa coincidência, se explorada, mostra que a teoria das classes de Serre generaliza, em certo sentido, a teoria da localização. 


\section{\$3. ESPACSOS NILPOTENTES}

Jä tivemos oportunidade de mencionar, na introdução deste trabalho, a importância dos espaços nilpotentes. Daremos agora uma definição precisa desses espaços e mencionamos al guns exemplos que mostram ser a categoria por eles constituida bem mais ampla do que a formada pelos C.W. complexos simplesmente conexos. Fazemos, em seguida, um resumo das pro priedades desses espaços que iremos utilizar no decorrer do trabalho.

3.3.0.DEFINICAO. Diremos que un C.W. complexo X é nilpotente se for conexo e se a ação de $\pi_{1} X$ em $\pi_{j} X$ for, para $j \geq 2$, nilpotente.

Decorre, imediatamente, dessa definição que todo C.W. complexo conexo e simplesmente conexo $\vec{e}$ um espaço nilpoten te.

E bem conhecido que se $x$ è um espaço de Hopf. $\pi_{1} X$ è abe Iiano e opera trivialmente em $\pi_{j} x, j \geq 1$. Consequentemente, todo espaço de Hopf conexo è nilpotente. Em particular, os grupos de Lie conexos são espaços nilpotentes.

No capitulo $\mathrm{V}$, provaremos que se $\mathrm{X} \overrightarrow{\mathrm{e}}$ um espaço nilpoten te $\mathrm{e} \overrightarrow{\mathrm{X}} \rightarrow \mathrm{X} \overrightarrow{\mathrm{e}}$ um recobrimento qualquer de $\mathrm{X}$ então $\overline{\mathrm{X}}$ è nilpotente. 
Uma forma muito ütil de caracterizar os espaços nilpotente è dada pelo seguinte

3.3.2 TEOREMA. Seja X um C.W. complexo conexo. Uma condição necessäria e suficiente para que X seja um espaço nilpotente é que $\pi_{1} X$ seja um grupo nilpotente e que sua ação nos grupos de ho mologia do recobrimento universal de $X$ seja nilpotente.

\section{DEMONSTRAÇÃO}

Ver pảginas 70 e 71 de $[H, M, R]$.

Exibimos agora um exemplo, que nos serä ûtil mais tarde, de um espaço que não $\vec{e}$ nilpotente.

3.3.3 EXEMPLO. $\operatorname{IRP}^{2}$ não è nilpotente.

Consideremos o recobrimento universal $\mathrm{S}^{2} \rightarrow \mathbb{R P}^{2}$. Bas ta, pelo teorema 3.3 .2 acima, provar que a ação $\omega$ de $\pi_{1} \operatorname{IRP}^{2}=$ $=\mathbb{Z} / 2 \mathbb{Z}$ em $\mathrm{H}_{2}\left(\mathrm{~S}^{2}\right)=\mathbb{Z}$ não é nilpotente.

Utilizando-se o fato de que a aplicação antípoda em uma esfera de dimensão par tem grau -1 verifica-se imediatamen te que

$$
\begin{aligned}
& \omega(0) \cdot x=x \\
& \omega(1) \cdot x=-x
\end{aligned}
$$

e portanto

$$
\Gamma_{\omega}^{2} \mathbb{Z}=\operatorname{gp}(\omega(1) \cdot x-x=-2 x)=2 \mathbb{Z}
$$


Uma simples indução nos leva a

$$
\Gamma_{\omega}^{n_{\mathbb{Z}}}=2^{n-1_{\mathbb{Z}}}
$$

e portanto w não è nilpotente.

E claro que o raciocinio acima se generaliza de forma a nos permitir mostrar que se $n \vec{e}$ par $\operatorname{RP}^{n}$ não $\vec{e}$ nilpotente.

$\mathrm{Na}$ realidade a demonstração acima nos mostra que $\mathrm{RP}^{2}$ não $\vec{e}$ nem mesmo homologicamente nilpotente. (Para a definição de homologicamente nilpotente ver 5.3 .7 , comentärio 1 ).

Embora não se vâ utilizä-la neste trabalho, $\ddot{e}$ oportuno alertar o leitor para o fato de que os espaços nilpotentes admitem uma caracterização mais geomêtrica.

3.3.4 TEOREMA. Seja X um C.W. complexo conexo. Então X ènil potente se e somente se o seu sistema de Postnikov admite un refinamento principal.

Concluiremos o parägrafo com algumas definições e resul tados que utilizaremos na demonstração do lema 5.3 .3 do capitulo V. A referência para esses töpicos $\vec{e}[H] 5^{\circ}$ 3.3.5 DEFINICRO. Seja $\mathrm{F} \longrightarrow \mathrm{E} \longrightarrow \mathrm{B}$ uma fibração com todos os espaços conexos. Dixemos que essa fibração $\vec{e}$ nilpotente se $\pi_{1}$ E operar nilpotentemente nos grupos de homotopia de $F$ (inclusive $\pi_{1} F$ ) e que ela $\vec{e}$ quase-nilpotente se $\pi_{1} B$ operar nilpotentemente nos grupos de homologia de $F$. 
Esses dois conceitos estão relacionados pelo seguinte 3.3.6 TEOREMA. Seja $F \longrightarrow E \rightarrow B$ uma fibração com todos os espaços conexos. Essa fibração ê nilpotente se e somente se ela for quase-nilpotente e F for nilpotente.

Este teorema $\ddot{e}$ o corolärio 2.2 de $\left[\mathrm{H}_{5}{ }^{\circ}\right.$

O pröximo teorema $\vec{e}$ um resultado essencial para a demons tração do lema 5.3 .3$.

3.3 .7 TEOREMA. Seja $\mathrm{F} \longrightarrow \mathrm{E} \rightarrow \mathrm{B}$ uma fibração quase nilpotente sobre a qual age o grupo G. Então

(a) Se G age nilpotentemente em $\mathrm{H}_{\mathrm{p}} \mathrm{B}, \mathrm{p} \leq \mathrm{P}$, e em $\mathrm{H}_{\mathrm{q}} \mathrm{F}, \mathrm{q} \leq \mathrm{Q}$, então G age nilpotentemente em $H_{n} E, n \leq N=\min (P, Q)$.

(b) Se G age nilpotentemente em $\mathrm{H}_{\mathrm{p}} \mathrm{B}, \mathrm{p} \leq \mathrm{P}$, em em $\mathrm{H}_{\mathrm{n}} \mathrm{E}, \mathrm{n} \leq \mathrm{N}$, então $G$ age nilpotentemente em $H_{q} F, q \leq Q=\min (P-1, N)$.

(c) Se G age nilpotentemente em $\mathrm{H}_{\mathrm{q}} \mathrm{F}, \mathrm{q} \leq \mathrm{Q}$, e em $\mathrm{H}_{\mathrm{n}} \mathrm{E}, \mathrm{n} \leq \mathrm{N}$, então $G$ age nilpotentemente em $H_{p} B, p \leq P=\min (Q+1, N)$. 

CAPITULO IV

ALGUNS RESULTADOS ALGEBRICOS RELATIVOS

A (CO)HOMOLOGIA DE GRUPOS MŌDULO C

§. INTRODUÇÃO

Concentramos neste capítulo todos os resultados referentes à (co)homologia de grupos módulo c que obtivemos neste traba1ho. Esses resultados, ao lado de seu interesse pröprio, serão cruciais ao desenvolvimento do capítu$10 \mathrm{~V}$.

O conceito de classe de Serre generalizada, introduzi do por Hilton, Mislin e Roitberg ( $\operatorname{ver}[H, M, R])$, desempenha rá um papel fundamental em tudo que faremos. Todos os nos sos resultados envolverão uma classe $C$ que será sempre su posta prōpria e acîclica.

E bom que se frise que não tratamos a homologia e a cohomologia em pé de igualdade. Apenas com relação à pri- 
meira procuramos ser mais completos. Na realidade, um uni co resultado se refere explicitamente à cohomologia e para obtê-10 tivemos que nos restringir aos grupos finitamente gerados.

As principais referências para este capitulo são $[H, M, R]$ e $[H, R]$. Tambëm são de utilidade $[H]_{1}$ e $[H]_{2}$. Embo ra em [S] só se considere classes de grupos abelianos a sua consulta poderä ser proveitosa. No que diz respeito a questões gerais de Algebra Homolögica, [H,S] e [M] säo boas referências.

No decorrer do capítulo utilizamos livremente as nota ções usuais da Âlgêbra Homolögica. Em particular, indicamos por $\mathbb{Z}[\pi]$ o anel de grupo do grupo $\pi$ e por $I[\pi]$ o seu ideal de aumentação. 
$\S 1$. HOMOLOGIA

Neste parägrafo e no pröximo c representarä sempre uma classe de Serre própria e acíclica de grupos nilpotentes.

4.1.0. LEMA Sejam A e B grupos abelianos com B em C. Se A for finitamente gerado ou se a classe C for completa en tก̃o:

i) $B \otimes A \in C$.

ii) $\operatorname{Tor}(B, A) \in C$.

DEMONSTRAÇÃO de i)

Seja $K \stackrel{\mu}{\longrightarrow}>L \stackrel{\varepsilon}{\longrightarrow}>$ A uma apresentação 1ivre de A.

Multiplicando tensorialmente por B obtemos a sequência exata

$$
\mathrm{B} \otimes \mathrm{K} \stackrel{1 \otimes \mu}{\longrightarrow}>\mathrm{B} \otimes \mathrm{L} \stackrel{1 \otimes \varepsilon}{\longrightarrow}>\mathrm{B} \otimes \mathrm{A} \longrightarrow \mathrm{\longrightarrow}
$$

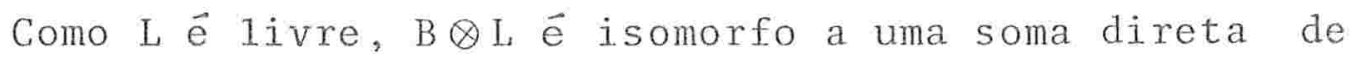
cöpias de B.

Se a classe C for completa é então öbvio que B $\otimes L \in C$. Caso conträrio, teremos, por hipötese, que A e B são finitamente gerados e portanto podemos construir a apresentação

$$
\mathrm{O} \longrightarrow \mathrm{K} \longrightarrow \mathrm{L} \longrightarrow \mathrm{A} \longrightarrow \mathrm{O}
$$


com L finitamente gerado o que também implica que $B \otimes L \in C$.

0 fato de $B \otimes L$ estar em $C$ e $I \otimes \varepsilon$ ser epimorfismo nos permite concluir que $B \otimes A \in C$ o que demonstra i).

DEMONSTRAÇAO de ii)

A demonstração é na realidade un corolärio da demonstração de i). De fato, consideremos novamente a sequência exata

$B \otimes K \stackrel{1 \otimes \mu}{\longrightarrow}>B \otimes L \stackrel{1 \otimes \varepsilon}{-}>B \otimes A \longrightarrow \longrightarrow 0$

O mesmo argumento que usamos acima para provar que $B \otimes L$ está em $C$ mostra que $B \otimes K$ pertence a $C$. E claro então que ker $1 \otimes \mu$ tambëm estä em $C$ o que conclui a demonstração pois $\operatorname{Tor}(A, B)=\operatorname{ker} 1 \otimes \mu$.

4.1.1. TEOREMA Sejam $\pi$ um grupo (não necessariamente nil potente) tal que $H_{i} \pi \in C, i \geq 1$, e A um grupo abeliano munido de uma estrutura de $Z[\pi]$ módulo à direita induzida por uma ação nilpotente $\omega$ de $\pi$ em A. Se A for finitamente gerado (como grupo) ou $C$ completa então $H_{i}(\pi, A) \in C$ para todo inteiro $i \geq 1$.

\section{DEMONSTRAÇAO}

Serä feita por indução sobre a classe de nilpotência da ação $\omega$.

1) Prova de que o teorema ë verdadeiro quando a ação 
$\overrightarrow{\mathrm{e}}$ trivial (classe $(\omega)=1$ ).

Nesse caso o teorema dos coeficientes universais nos fornece a sequência exata

$$
\mathrm{O} \longrightarrow \mathrm{H}_{i} \pi \otimes \mathrm{A} \longrightarrow \mathrm{H}_{i}(\pi, \mathrm{A}) \longrightarrow \operatorname{Tor}\left(\mathrm{H}_{\mathrm{i}-1} \pi, \mathrm{A}\right) \longrightarrow 0
$$

Para obtermos a tese resta provar então que $H_{i} \pi \otimes A \quad$ e $\operatorname{Tor}\left(\mathrm{H}_{i-1} \pi, A\right)$ estão em $C$ quando $i \geq 1$.

Por hipötese, para todo $i \geq 1, H_{i} \pi \in C$ e, portanto, pelo lema 4.1.0., $\mathrm{H}_{\mathbf{i}} \pi \otimes \mathrm{A} \in \mathrm{C}$. O mesmo argumento mostra que para $i \geq 2 \operatorname{Tor}\left(H_{i-1} \pi, A\right)$ está em $C$. O caso $i=1$ é trivial pois $\operatorname{Tor}\left(\mathrm{H}_{0} \pi, \mathrm{A}\right)=\operatorname{Tor}(\mathrm{Z}, \mathrm{A})=0$.

A ültima igualdade decorre do fato de $Z$ ser livre.

2) Provaremos agora que a suposição de que o teorema é verdadeiro para ações de classe menor ou igual a n-1 impli ca em sua validade para ações de classe n.

Consideramos a sequência exata de $\mathrm{Z}[\pi]$ môdulos

$$
\mathrm{O} \longrightarrow \mathrm{T} \longrightarrow \mathrm{A} \longrightarrow \mathrm{A} / \mathrm{I} \longrightarrow \mathrm{O}
$$

onde $\Gamma=\Gamma_{\omega}^{2} \mathrm{~A}$.

Essa sequência induz uma sequência exata longa de homologia da qual destacamos, para anälise, o trecho

$$
\mathrm{H}_{i}(\pi, \Gamma) \rightarrow \mathrm{H}_{i}(\pi, \mathrm{A}) \longrightarrow \mathrm{H}_{i}(\pi, \mathrm{A} / \Gamma)
$$

onde $i \geq 1$.

Como as ações de $\pi$ em $\Gamma$ e A/ $\Gamma$ tem classe n-1 e 1, 
respectivamente, a hipótese de indução nos diz que os gru pos $H_{i}(\pi, \Gamma)$ e $H_{i}(\pi, A / \Gamma)$ estão em C. Esse fato e a exatidão da sequência a que os grupos pertencem nos garantem (confronte a propriedade 3.2.10.) que $H_{i}(\pi, A)$ estå em C. O argumento de indução estả então completo e o teorema demonstrado.

\subsubsection{OBSERVACOES}

1) Ao lado de enfatizarmos o fato de não havermos suposto o grupo $\pi$ nilpotente queremos lembrar ao leitor que, como somente consideramos classes acíclicas e claro que se $\pi \in C$ então $H_{i} \pi \in C, i \geq 1$. Nas aplicações do teorema 4.1.1. (e dos outros resultados deste capítulo) à topologia, nos capítulos V e VI, a condição $\pi \in C$ estará (para uma conveniente escolha da classe C) sempre satisfeita por estarmos trabalhando com espaços nilpotentes. Existem, no entanto, situações importantes nas quais apenas a condição $H_{i} \pi \in C$, $i \geq 1$, se verifica $(5.3 .7$. , comentärio 1$)$.

2) Vimos acima que $\pi \in C \Rightarrow H_{i}(\pi, A) \in C, i \geq I$.

Serä a restrição izI essencial? A igualdade $H_{0}(\pi, A)=A$, que se verifica sempre que a ação de $\pi$ em A è trivial, mos tra que sim. Na realidade, prova-se (confronte teorema 5.6. de $[H . M . R]$ ) que se as hipöteses do teorema 4.1.1. estive- 
rem verificadas e $\pi \in C$ então $H_{0}(\pi, A) \in C \Leftrightarrow A \in C$. Esse fato dả ao teorema 4.1.4. um caräter até certo ponto surpreendente.

Provaremos agora un teorema que admite un corolärio (corolärio 4.1.4.) que nos será de grande valia no capítulo V. As hi póteses são (a não ser pelo fato de agora exigirmos que $H_{i} \pi \in C$ apenas para $i=1$ ) as mesmas do teorema 4.1 .1 .

4.1.3. TEOREMA Seja $\pi$ um grupo (não necessariamente nilpotente) tal que o seu abelianizado $\pi_{\mathrm{ab}}$ esteja em $\mathrm{C}$ e, A

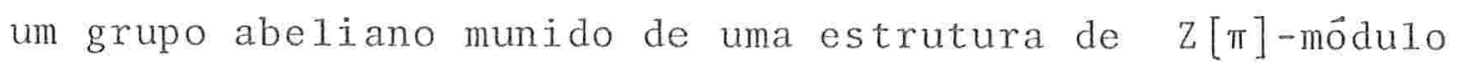
à direita induzida por uma ação nilpotente $\omega$ de $\pi$ em A. Se A for finitamente gerado ou C completa podemos afirmar que :

i) $A \otimes I[\pi] \in C$

ii) $A \cdot I[\pi]^{i}=\Gamma_{\omega}^{i+1} A \in C$ para todo inteiro $i z 1$.

DEMONSTRAÇÃO de i)

Por indução sobre a classe de nilpotência de $\omega$.

1) Se a ação $\omega$ for trivial sabemos que

$$
\begin{aligned}
& A \otimes I[\pi]=H_{1}(\pi, A) \\
& H_{1}(\pi, A)=H_{1} \pi \otimes A .
\end{aligned}
$$

A primeira igualdade ê bastante conhecida (ver, por 
exemp1o, pag. 192 de $[H, S])$ e a segunda $\vec{e}$, como jă vimos na demonstração do teorema 4.1.1., consequência do teorema dos coeficientes universais. Para concluir a demonstra ção resta observar que como $H_{1} \pi$ estä em $C\left(H_{1} \pi=\pi a b^{\prime}\right)$, pe lo lema 4.1.0., $H_{1} \pi \otimes A$ tambëm estâ em $C$.

2) Suponhamos o resultado vâlido para açöes de classe $\leq n-1$ e consideremos a sequência exata

$$
\mathrm{O} \longrightarrow \mathrm{P} \rightarrow \mathrm{A} \longrightarrow \mathrm{A} / \mathrm{I} \longrightarrow \mathrm{O} \text {. }
$$

Multiplicando tensorialmente por $I[\pi]$ obtemos a sequência (tambẻm exata)

$$
\Gamma \otimes I[\pi] \rightarrow A \otimes I[\pi] \rightarrow A / \Gamma \otimes I[\pi] \rightarrow 0
$$

A exatidão da sequência e a hipótese de indução (que nos garante que $\Gamma \otimes I[\pi]$ e $A / \Gamma \otimes I[\pi]$ estão em $C$ ) nos levam então a concluir que $A \otimes I[\pi] \in C$, o que completa o argumento de indução.

DEMONSTRAÇAO de ii)

Como para todo $j \geq 1$ A.I $[\pi]^{j} \vec{e}$ um subgrupo de A.I $[\pi]$, bas ta mostrar que A.I $[\pi]$ estä em C.

Consideremos a aplicação

$$
A \times I[\pi] \stackrel{H}{\longrightarrow}>A . I[\pi]
$$

definida por $\mu(a, x)=a \cdot x$.

Podemos então construir o diagrama comutativo 


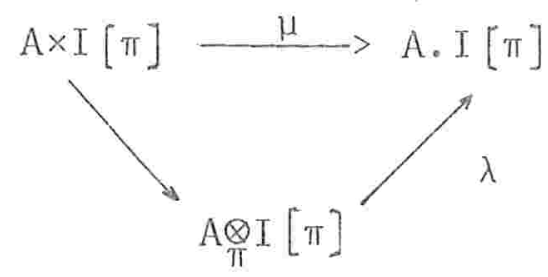

onde $\lambda(a \otimes x)=a \cdot x$.

O fato de $\lambda$ ser um epimorfismo (A.I [ $\pi]$ è gerado por elementos da forma ax) e de $A_{\pi}^{\otimes}[\pi] \in C$ (item i) do teorema) nos mostra que A.I $[\pi] \in C$.

4.1.4. COROLARIO Sejam $\pi$ um grupo tal que $\pi_{a b} \in c$ e A um grupo abeliano no qual $\pi$ age nilpotentemente. Se A for fi nitamente gerado ou $\mathcal{C}$ for completa então a projeção $A \stackrel{\varepsilon}{\longrightarrow}(A)_{\pi}$, onde $(A)_{\pi}=A / \Gamma$ é uma $C$ bijeção.

\section{DEMONSTRAÇÃO}

Como $\varepsilon$ é uma sobrejeção basta observar que ker $\varepsilon=\Gamma=A \cdot I[\pi]$ estä, pelo item ii) do teorema acima, em $C$.

\subsubsection{COMENTARIOS}

1) $\mathrm{Na}$ demonstração do teorema 4.1.1. usamos de maneira decisiva o fato da ação $\omega$ de $\pi$ em A ser nilpotente. Em bora essa hipötese seja aparentemente indispensảvel, não possuimos um contra-exemplo para o teorema no caso dela não estar satisfeita.

2) Considerando-se a classe $\mathrm{C}$ dos grupos nilpotentes de torção e um grupo $\pi$ com dois elementos (que indicare- 
mos por 1 e - - l) consegue-se facilmente um contra-exemplo para o teorema 4.1.3. e para o corolärio 4.1.4. no caso da ação não ser nilpotente. De fato, suponhamos que $\pi$ ope re no grupo $\mathbb{Z}$ dos inteiros atravës de uma ação w tal que

$$
\omega(-1) \cdot n=-n
$$

(essa ê exatamente a açăo de $\pi_{1}\left(\mathbb{R} P^{2}\right)$ em $\mathrm{H}_{2}\left(\mathbb{R} \mathrm{P}^{2}\right)$ considera da no capítulo III).

As igualdades

$$
I[\pi]^{n-1} \cdot z=\Gamma_{\omega}^{n}(z)=2^{n-1} z
$$

mostram, como jă vimos em 3.3.3., que a açäo w näo é nilpotente.

Como $I[\pi] . Z=2 Z$ não pertence a $C$ por não ser um grupo de torção, temos un contra-exemplo para p item ii) do teo rema e para seu corolärio (se considerarmos a projeção $\mathrm{ZL} \stackrel{\varepsilon}{\longrightarrow} \mathrm{Z} / \mathrm{I}[\pi] \cdot \mathrm{Z}, \operatorname{ker} \varepsilon=I[\pi] \mathrm{Z}$ \& e portanto $\varepsilon$ não $\overrightarrow{\mathrm{e}} \mathrm{c}-$ in jetora).

Por ser o homomorfismo

$$
\underset{\pi}{Z \otimes I}[\pi] \longrightarrow Z \cdot I[\pi]
$$

sobrejetor, concluimos que $Z \otimes I[\pi]$ näo pertence a $C$ e por tanto tambëm o item i) do teorema näo se verifica.

3) A hipötese de que o grupo A do corolärio 4.1.3. se ja abeliano é imprescindivel como podemos ver pelo seguin te exemplo: Seja C a classe trivial (classe C constituida 
por grupos com um único elemento), $\pi$ um grupo de $C$ e supo nhamos que A não seja abeliano. Nessas condições $[A, A] \vec{e}$ não trivial $\mathrm{A} / \Gamma=\mathrm{A}_{\mathrm{ab}}$. Essa igualdade é consequência do fato de $\pi$ ser trivial e da pröpria definição de $\Gamma=\Gamma_{\omega}^{2} \mathrm{~A}$ no caso de A não ser abeliano (confronte 3.1.3.). E claro então que a projeção $A \stackrel{\varepsilon}{\longrightarrow} A / \Gamma$ não pode ser uma $C$-bijeção pois ker $\varepsilon=[A, A] \& C$.

Finalizamos o parágrafo com uma proposição que usamos no capitulo $\mathrm{V}$ para mostrar que os resultados $1 \vec{a}$ obtidos não exigem que se utilize homologia com coeficientes inteiros.

4.1.6. PROPOSICAO Sejam B $1, B_{2}$, A grupos abelianos e $\mathrm{B}_{1} \stackrel{\lambda}{\rightarrow} \mathrm{B}_{2}$ uma $\mathrm{C}$-bijeção. Se A for finitamente gerado ou se a classe C for completa então os homomorfismos

$$
\begin{gathered}
\mathrm{B}_{1} \otimes \mathrm{A} \stackrel{\lambda \otimes 1}{\rightarrow} \mathrm{B}_{2} \otimes \mathrm{A} \\
\operatorname{Tor}\left(\mathrm{B}_{1}, \mathrm{~A}\right) \stackrel{\lambda_{*}}{\rightarrow} \operatorname{Tor}\left(\mathrm{B}_{2}, \mathrm{~A}\right)
\end{gathered}
$$

são tambêm C-bijeções.

DEMONSTRAÇAOO

Consideramos o homomorfismo $\lambda$ como composto de um epi morfismo com um monomorfismo como o indicado no diagrama comutativo

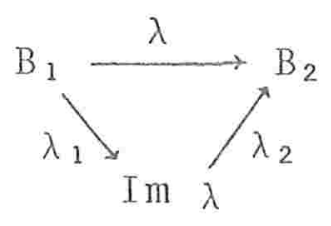


onde $\lambda_{1}$ è o pröprio homomorfismo $\lambda$ com o contra-domínio al terado para Im $\lambda$ e $\lambda_{2}$ é a inclusão.

E claro que ker $\lambda_{1}=\operatorname{ker} \lambda$ e coker $\lambda_{2}=\operatorname{coker} \lambda$ e por tanto as sequências

(1) $0 \longrightarrow \operatorname{ker} \lambda \stackrel{i_{1}}{\longrightarrow} B_{1} \stackrel{\lambda_{1}}{\longrightarrow}$ Im $\lambda \longrightarrow 0$

(2) $0 \longrightarrow$ Im $\lambda \stackrel{\lambda_{2}}{\longrightarrow} B_{2} \stackrel{p_{2}}{\longrightarrow} \operatorname{coker} \lambda \longrightarrow 0$

onde $i_{1}$ e $p_{2}$ tem os significados öbvios (inclusão e proje ção) são exatas. Utilizaremos mais adiante o fato (decorrente de $B_{1} \stackrel{\lambda}{\rightarrow} B_{2}$ ser una $C-$ bijeção) de ker $\lambda$ e coker $\lambda$ estarem em C.

A decomposição acima nos permite construir os diagramas comutativos

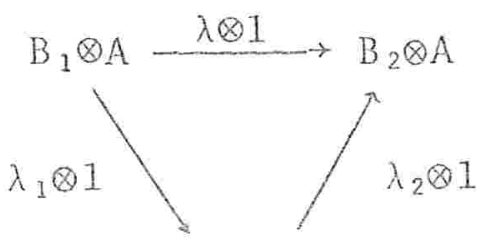

$\operatorname{Im} \lambda \otimes A$

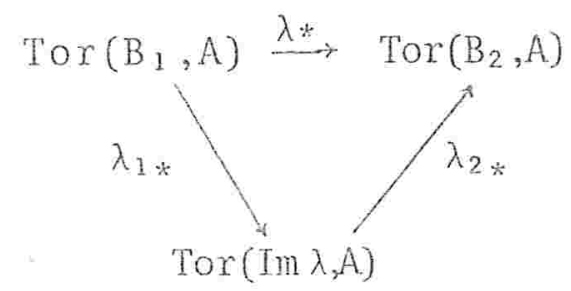

$\operatorname{Tor}(\operatorname{Im} \lambda, \mathrm{A})$

Uma vez que sabemos que a composiçäo de c-bijeções é uma $C$-bijeção basta provar que $\lambda_{1} \otimes 1, \lambda_{2} \otimes 1, \lambda_{1 *}, \lambda_{2 *}$ säo c-bijeçōes e teremos a tese.

Examinemos a sequência exata

$\mathrm{O} \longrightarrow \operatorname{Tor}(\operatorname{Im} \lambda, \mathrm{A}) \stackrel{\lambda_{2 *}}{\longrightarrow} \operatorname{Tor}\left(\mathrm{B}_{2}, \mathrm{~A}\right) \stackrel{\mathrm{p}_{2 *}}{\longrightarrow} \operatorname{Tor}(\operatorname{coker} \lambda, \mathrm{A}) \stackrel{\omega}{\longrightarrow}$ $\stackrel{\omega}{\rightarrow}$ Im $\lambda \otimes A \stackrel{\lambda_{2} \otimes 1}{\rightarrow} B_{2} \otimes A \stackrel{p_{2} \otimes 1}{\longrightarrow}$ coker $\lambda \otimes A \longrightarrow 0$ 
Pelo Lema 4.1.0., Tor $(\operatorname{coker} \lambda, A)$ e coker $\lambda \otimes A$ estão em C e, portanto, $\lambda_{2 *}$ e $\lambda_{2} \otimes 1$ são C-bijeções. Para provar que $\lambda_{1 *}$ e $\lambda_{1} 1$ são $C$-bijeções utilizamos a sequência exata in duzida por (1) . 


\section{$\S 2$. COHOMOLOGIA}

Examinaremos agora quais resultados podemos transpor tar, a partir do que fizemos no parägrafo anterior, para a cohomologia. Nossa intenção não é, como jä foi dito na introdução a este capitulo, fazer um estudo aprofundado da situação cohomolögica, mas, tão somente, utilizar o teorema dos coeficientes universais como ponte entre as teorias homológica e cohomológica. Essa ë a razão de não tentamos demonstrar resultados "anälogos" a 4.1 .3 . e a 4.1.4. embora seja provävel que, com as devidas adaptações eles possam ser enunciados.

Começaremos com o seguinte

4.2.0. LEMA Sejam A e B grupos abelianos finitamente gerados. Se $A$ pertencer a $C$, $\operatorname{Hom}(A, B)$ e Ext $(A, B)$ também pertencerão a $C$.

DEMONSTRAÇAOO

Sendo A um grupo abeliano e finitamente gerado demons tra-se $[H, S]$, sem dificuldade a existência dos seguintes isomorfismos:

$$
\begin{array}{ll}
\operatorname{Hom}(A, \mathbb{Z}) \rightarrow \mathrm{LA}, & \operatorname{Hom}(\mathrm{A}, \mathbb{Z} / \mathrm{mZ}) \mp \mathrm{A} / \mathrm{mA} \\
\operatorname{Ext}(\mathrm{A}, \mathbb{Z}) \rightarrow \mathrm{TA}, & \operatorname{Ext}(\mathrm{A}, \mathbb{Z} / \mathrm{mZ}) \approx \mathrm{TA} / \mathrm{mTA}
\end{array}
$$

onde LA e TA indicam, respectivamente, as partes livre e 
de torção do grupo A. E claro então que basta que A esteja em $C$ para que qualquer dos grupos que figuram nos isomorfismos acima esteja em $C$.

Utilizando os isomorfismos

$$
\begin{aligned}
& \operatorname{Hom}\left(A, \stackrel{n}{\oplus} B_{i}\right) \underset{i=1}{\stackrel{n}{\oplus}} \operatorname{Hom}\left(A, B_{i}\right) \\
& \operatorname{Ext}\left(A, \oplus_{i=1}^{\oplus} B_{i}\right) \approx \stackrel{\oplus}{\oplus}=1^{n} \operatorname{Ext}\left(A, B_{i}\right),
\end{aligned}
$$

lembrando que $B$ é isomorfo a uma soma direta finita de grupos cíclicos (B è finitamente gerado) levando em conta o que vimos acima concluimos que $\operatorname{Hom}(A, B)$ e $\operatorname{Ext}(A, B)$ são somas diretas finitas de grupos pertencentes a $C$ e portan to tambẻm estão em $C$.

Existe um contra-exemplo bastante simples para o lema acima no caso do grupo A não ser finitamente gerado. Para construi-1o consideramos a classe C dos grupos nilpotentes de torção e definimos o grupo A pela igualdade $A=\underset{j \in N_{+}}{\oplus} A_{j}$ onde $A_{j}=\mathbb{Z} / j \mathbb{Z}$ e $N_{+} \vec{e}$ o conjunto dos números naturais estritamente positivos.

E claro que A é um grupo de torção e portanto está em c. Tambẻm não hả dủvida que A não ê finitamente gerado.

Tomamos para B o grupo $\mathbb{Z}$ dos inteiros utilizando os isomorfismos

$$
\left.\operatorname{Ext} \underset{j \in N_{+}}{\oplus} A_{j}, \mathbb{Z}\right) \sim \underset{j \in N_{+}}{\pi} \operatorname{Ext}\left(A_{j}, \mathbb{Z}\right)
$$




$$
\operatorname{Ext}\left(A_{j}, \mathbb{Z}\right) \sim A_{j}
$$

vemos que Ext $(A, B) \stackrel{\rightarrow}{j \in N_{+}} A_{j}$.

Portanto Ext (A,B) não estä em $\mathcal{C}$ por não ser um grupo de torção. Temos assim o contra-exemplo.

Demonstraremos agora um resultado que $\vec{e}$ o anảlogo, pa ra a cohomologia, do teorema 4.1.1. do parägrafo anterior.

4.2.1. TEOREMA Seja A um grupo abeliano finitamente gera do e $\pi$ un grupo tal que se $i \geq 1 H_{i} \pi \vec{e}$ finitamente gerado e pertence a $C$. Suporemos A munido de uma estrutura de $\mathbb{Z}[\pi]$ módulo à esquerda induzida por uma ação nilpotente $\omega$ de $\pi$ em A. Nessas condiçōes $H^{i}(\pi, A) \in C, i \geq 1$.

DEMONSTRACAO

Por indução sobre a classe de nilpotência de $\omega$.

1) A ação w è trivial.

Consideramos a sequência exata

$$
0 \longrightarrow \operatorname{Ext}\left(H_{i-1} \pi, A\right) \longrightarrow H^{i}(\pi, A) \longrightarrow \operatorname{Hom}\left(H_{i} \pi, A\right) \longrightarrow 0
$$

dada pelo teorema dos coeficientes universais.

Como o lema 4.2.0. e a igualdade $\operatorname{Ext}\left(H_{0} \pi, A\right)=\operatorname{Ext}(\mathbb{Z}, A)=0$ nos garantem que para $i \geq 1$ Ext $\left(H_{i-1} \pi, A\right)$ e $\operatorname{Hom}\left(H_{i} \pi, A\right)$ estão em $C$, temos que $H^{i}(\pi, A)$ tambëm estä em $C$. 
2) Provamos que a suposição de que o teorema é vâlido, para ações de classe $\leq n-1$ implica na sua validade para ações de classe n.

Escreve-se a sequência exata longa de cohomologia induzida pela sequência exata de $\mathbb{Z}[\pi]$ módulos

$$
\mathrm{O} \longrightarrow \mathrm{\Gamma} \longrightarrow \mathrm{A} \longrightarrow \mathrm{A} / \mathrm{\Gamma} \longrightarrow \mathrm{O} \quad\left(\Gamma=\Gamma_{\omega}^{2} \mathrm{~A}\right)
$$

e argumenta-se como no final da demonstração do teorema 4.1 .1

E claro que, a exemplo do que ocorre com a homologia, aqui também não podemos pretender que, nas hipöteses do teorema, $H^{0}(\pi, A) \in C$ (confronte 4.1.2., observação 2).

o próximo resultado, com o qual encerramos este parágrafo, mostrarả a sua utilidade ao tratarmos da cohomologia no pröximo capítulo.

4.2.2. PROPOSIÇAO Sejam $A_{1}, A_{2}$ e B grupos abelianos fini tamente gerados e $A_{1} \stackrel{\phi}{\longrightarrow} A_{2}$ uma $C$-bijeção. Nessas condições os homomorfismos

$$
\begin{aligned}
& \operatorname{Hom}\left(A_{2}, B\right) \longrightarrow \operatorname{Hom}\left(A_{1}, B\right) \\
& \operatorname{Ext}\left(A_{2}, B\right) \longrightarrow \operatorname{Ext}\left(A_{1}, B\right)
\end{aligned}
$$

induzidos por $\phi$, são também C-bijeções.

DEMONSTRAÇAOO

E, com as devidas adaptações, uma repetição da demons 
tração da proposição 4.1.6. e portanto nos satisfaremos en apresentä-1a esquematicamente.

A fatoração de $\phi$ indicada no diagrama comutativo

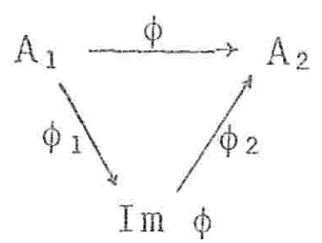

nos permite construir os diagramas abaixo, tambëm comutativos

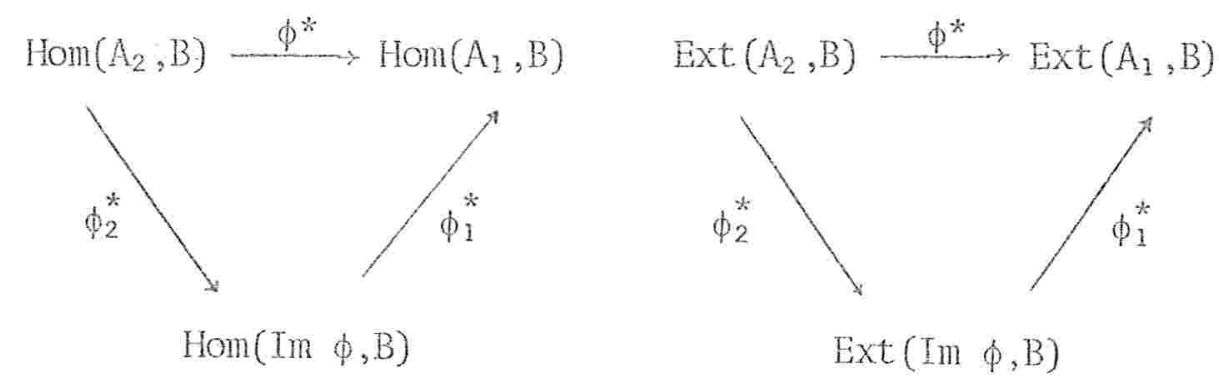

(Estamos aqui cometendo um abuso de notação, usual na Algebra Homolögica, dando a homomorfismos diferentes o mesmo nome!)

E claro que se provarmos que em cada un dos diagramas acima $\phi_{1}^{*}$ e $\phi_{2}^{*}$ são C-bijeções teremos a tese.

Demonstramos isso considerando as sequências

1) $0 \longrightarrow \operatorname{ker} \phi \stackrel{i_{1}}{\rightarrow} A_{1} \stackrel{\phi 1}{\longrightarrow} \operatorname{Im} \phi \longrightarrow 0$

2) $0 \longrightarrow \operatorname{Im~} \phi \stackrel{\phi_{2}}{\rightarrow} \mathrm{A}_{2} \stackrel{\mathrm{p}_{2}}{\rightarrow}$ coker $\phi \rightarrow 0$

e examinando as sequências exatas longas obtidas pela aplicação do Euntor Hom a elas levando em conta o fato de, 
peIo Lema 4.2.0. Hom (ker $\phi, B)$, Hom (coker $\phi, B), \operatorname{Ext}(\operatorname{ker} \phi, B)$ e Ext(coker $\phi, B)$ estarem em C. 



\section{CAPITULO V}

HOMOLOGIA MÖDULO C DE RECOBRIMENTOS DE ESPAÇOS NILPOTENTES

$\S 0$. INTRODUÇÃO

De volta à Topologia, colheremos os frutos de nossa râa pida incursão pela Ảlgebra. Usando métodos elementares e sem complicações técnicas obteremos informações sobre os grupos de homologia de recobrimentos de espaços nilpotentes que nos permitirão, como exemplificamos no capítulo VI, desenvolver de uma forma nova, o programa de transportar, da categoria dos espaços simplesmente conexos para a categoria dos espaços nilpotentes, técnicas e resultados de re conhecida importância.

o objetivo dos parägrafos um e dois é comparax o comportamento, face a uma projeção de recobrimento, dos grupos de homotopia e homologia. Como é sabido, o que se verifica é que, apesar do comportamento dos grupos de homotopia ser 
muito bom, $\vec{e}$ muito difäcil prever o que acontece com a ho mologia.

o parägrafo três é mais 1ongo. Começa com um estudo das propriedades mais simples dos recobrimentos de espaços nilpotentes e culmina com o teorema 5.3.4. Esse teore ma e seus corolärios são os principais resultados do capí tulo e, provavelmente, de todo o trabalho.

No parägrafo quatro indicamos uma forma simples, mas que provavelmente não é a que dä melhores resultados, de transferir para o caso relativo o corolârio 5.3 .5 .

Isso nos permitirä, no capítulo VI, apresentar uma forma relativa do teorema de Hurewicz módulo C para espaços nilpotentes.

o quinto e ültimo parägrafo trata da cohomologia. o enfoque $\vec{e}$, de certa forma, superficial. Um tratamento mais aprofundado exigiria um desenvolvimento maior da parte a 1 gébrica estudada no capitulo anterior. As referências gerais para o capítulo são $[H, M, R],[H],[H, R]_{1},[H, R]_{2} \cdot 0-$ ferecemos, no texto, indicaçöes mais precisas. 
§1. ESPAÇOS DE RECOBRIMENTO: PROPRIEDADES HOMOTÓPICAS E DE LEVANTAMENTO

5.1.0. Seja X um C.W.complexo conexo e $\tilde{X} \rightarrow X$ um recobrimento qualquer de $\mathrm{X}$ na categoria dos espaços topoIögicos. E bem sabido (ver [W]) que, nessas condições, podemos dar a $\tilde{X}$, atravês de uma decomposição celular conveniente, uma estrutura de C.W.complexo, de forma que a aplicação $p$ seja celular. Tambêm è bastante conhecido o fato de que nem todo espaço topológico admite un recobrimento universal. Como esse ê um ponto bảsico para o desenvolvimento da teo ria, $\vec{e}$ usual exigirmos que os espaços considerados satisfa çam alguma condição suplementar (por exemplo, que sejam semi-localmente simplesmente conexos) que nos assegure a existência desses recobrimentos. Se nos restringirmos a espaços que admitem una estrutura de c.w.complexo, essa preocupação deixa de existir. A razão disso è que tais es paços são localmente conträteis. Essa é uma propriedade bastante forte e que permite um desenvolvimento completo da teoria dos recobrimentos. Um espaço localmente contrátil, alêm de ser localmente conexo por caminhos e semi-1ocalmente simplesmente conexo, possui muitas outras proprie dades agradáveis do ponto de vista da teoria da homotopia.

As considerações acima devem ter convencido o leitor 
de que na categoria dos c.w.complexos, na qual nos coloca mos neste trabalho, a teoria dos recobrimentos se ambienta com bastante naturalidade.

Passamos agora a descrever algumas propriedades homotô picas e de levantamento dos revestimentos.

Salvo menção em conträrio, estaremos supondo sempre que os espaços com que tratamos são conexos.

5.1.1. Consideremos um recobrimento $\tilde{x} \stackrel{p}{\rightarrow} x$ e dois pontos $\tilde{x}_{0}$ e $\tilde{x}_{0}^{\prime}$ de $p^{-1}\left(x_{0}\right)$. E un fato bem conhecido que

$$
\mathrm{p} * \pi_{1}\left(\tilde{X}, \tilde{x}_{0}\right) \text { e } p * \pi_{1}\left(\tilde{X}, \tilde{x}_{0}^{\prime}\right)
$$

são subgrupos conjugados de $\pi_{1}\left(X, X_{0}\right)$. Na rea]idade, podemos afirmar ainda mais: se $\pi_{0} \vec{e}$ um subgrupo de $\pi_{1}\left(X, x_{0}\right)$ conjugado a $\mathrm{p} * \pi_{1}\left(\widetilde{\mathrm{X}}, \tilde{\mathrm{x}}_{0}\right)$ então existe $\mathrm{x}_{0}^{\prime} \in \mathrm{p}^{-1}\left(\mathrm{x}_{0}\right)$ tal que $\mathrm{p} * \pi_{1}\left(\tilde{X}, \tilde{\mathrm{x}}_{0}^{1}\right)=\pi_{0}$.

Em face dessas propriedades ocorre-nos, naturalmente, colocar a questão de forma inversa, isto é, dado um espaço $x$ indagamos se existe um recobrimento $\tilde{x} \stackrel{p}{\rightarrow} x$ tal que $p * \pi_{1} \tilde{X}$ seja um subgrupo predeterminado de $\pi_{1} X$.

o principal resultado nessa direção é o seguinte

5.1.2. TEOREMA Dado o espaço $\left(X, x_{0}\right)$ fixemos uma classe de conjugação de subgrupos de $\pi_{1}\left(X, x_{0}\right)$ à qual pertence o grupo 
G. Existe um recobrimento $\tilde{x} \stackrel{p}{\longrightarrow} x$, e um ponto $\tilde{x}_{0} \in p^{-1}\left(x_{0}\right)$ tais que $\mathrm{p} * \pi_{1}\left(\tilde{\mathrm{X}}, \tilde{\mathrm{x}}_{0}\right)=\mathrm{G}$. O recobrimento $\tilde{\mathrm{X}} \stackrel{\mathrm{e}}{\rightarrow} \mathrm{X} \overrightarrow{\mathrm{e}}$, a menos de um homeomorfismo canônico, ünico.

DEMONSTRAÇÃO

A unicidade ê uma conseqtência imediata do teorema 5.1.4. abaixo. Para a demonstração da existência ver [M]. Uma outra característica notảvel das projeções de recobrimento é dada pelo

5.1.3. TEOREMA Seja $\tilde{\mathrm{X}} \stackrel{\mathrm{p}}{\rightarrow} \mathrm{X}$ um recobrimento qualquer. 0 homomorfismo $\pi_{j} \tilde{X} \stackrel{p *}{\longrightarrow} \pi_{j} X \widehat{e}$ um isomorfismo se $j>1$ e um mo nomorfismo se $j=1$.

Esse resultado vale em consequência das propriedades de levantamento (ünico) de caminhos e de homotopias que são características dos espaços de recobrimento. Para uma demonstração do teorema ver $|M|$.

No que se refere às propriedades de levantamento, o se guinte teorema è de fundamental importância.

5.1.4. TEOREMA Consideremos o recobrimento $\left(\tilde{X}, \tilde{x}_{0}\right) \stackrel{p}{\longrightarrow}\left(X, x_{0}\right)$ e uma aplicação contínua $\left(Y, y_{0}\right) \stackrel{f}{\longrightarrow}\left(X, X_{0}\right)$. Uma condição necessäria e suficiente para que exista uma aplicação con tínua $\left(Y, y_{0}\right) \stackrel{\tilde{f}}{\rightarrow}\left(\tilde{X}, \tilde{x}_{0}\right)$ tal que o diagrama abaixo seja 
comutativo è que $f * \pi_{1}\left(Y, y_{0}\right) \subset p * \pi_{1}\left(\tilde{X}, \tilde{x}_{0}\right)$.

A função $\tilde{f}$, quando, existe, $\ddot{\mathrm{e}}$ unica.

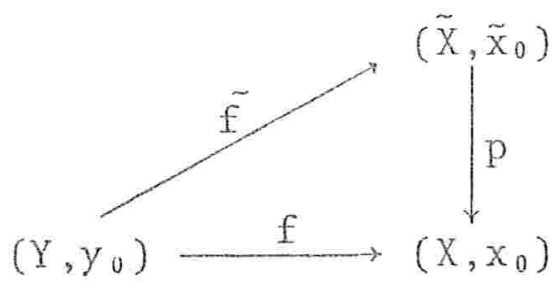

DEMONSTRACAAO

$\operatorname{Ver}|M|$ 。

Para finalizar enunciamos uma propriedade dos recobrimentos que nos serä ütil no pröximo parảgrafo.

5.1.5. Se $x_{1} \stackrel{p_{1}}{\rightarrow} x$ e $x_{2} \stackrel{p_{2}}{\rightarrow} x_{1}$ são recobrimentos então $x_{2} \frac{p_{1} \cdot p_{2}}{\rightarrow} x$ é um recobrimento.

A demonstração apoia-se no fato do espaço $x$ possuir um recobrimento universal (confronte [M] Ex. 6.5 pägina 161)。 
§2. ESPAÇOS DE RECOBRIMENTO: RELAÇAO ENTRE OS GRUPOS DE HOMOLOGIA DE UM ESPAÇO E DO SEU RECOBRIMENTO. SEQUEN CIA ESPECTRAL DE UM RECOBRIMENTO.

Um dos objetivos que tivemos em mente ao rememorarmos, no parägrafo anterior, alguns fatos bảsicos da teoria dos recobrimentos foi ressaltar a maneira simples mas profunda como se relacionam os conceitos de recobrimento e homotopia. Um dos resultados que enunciamos (teorema 5.1.3) mostra que, do ponto de vista estrito da homotopia (grupos de homotopia), um espaço e seu recobrimento são quase indistinguíveis.

Neste parágrafo transferimos o nosso interesse para a homologia, isto é, pretendemos examinar as relações en tre a homologia de um espaço e a do seu recobrimento.

Consideremos um recobrimento $\bar{X} \rightarrow X$. Se $\pi_{1} X$ for abe liano, as relaçöes $\pi_{1} \bar{X} \subset \pi_{1} X$ e $H_{1} X=\pi_{1} X /\left[\pi_{1} X, \pi_{1} X\right]$ mostram de imediato que, a exemplo do que acontece com os grupos fundamentais, temos uma inclusão $\mathrm{H}_{1} \overline{\mathrm{X}} \longrightarrow \mathrm{H}_{1} \mathrm{X}$ na homologia. Nas dimensöes mais altas, ou mesmo em dimensão um quando $\pi_{1} X$ não ë abeliano, podem surgir complicações.

Vejamos alguns casos concretos. 


\section{2 .0$. EXEMPLOS}

1) $\mathbb{R} \longrightarrow \mathrm{S}^{1}$

Os grupos de homologia são:

$$
\begin{aligned}
& H_{0}(\mathbb{R})=H_{0}\left(S^{1}\right)=\mathbb{Z}, \\
& H_{1}(\mathbb{R})=0, \quad H_{1}\left(S^{1}\right)=\mathbb{Z} e \\
& H_{j}(\mathbb{R})=H_{j}\left(S^{1}\right)=0, \quad j \geq 2 .
\end{aligned}
$$

Neste exemplo, os grupos de homologia do espaço $\mathrm{S}^{1}$ e de seu recobrimento $\mathbb{R}$ são, exceto em dimensão um, os mes mos. O homomorfismo $\mathrm{H}_{1}(\mathbb{R}) \longrightarrow \mathrm{H}_{1}\left(\mathrm{~S}^{1}\right)$, como $\mathrm{ja}$ se poderia prever pelo que dissemos acima, é uma inclusão.

2) $\mathbb{R}^{2} \longrightarrow S^{1} \times S^{1}$.

Vejamos os grupos de homologia nesse caso:

$$
\begin{aligned}
& \mathrm{H}_{0}\left(\mathbb{R}^{2}\right)=\mathrm{H}_{0}\left(\mathrm{~S}^{1} \times \mathrm{S}^{1}\right)=\mathbb{Z}, \\
& \mathrm{H}_{1}\left(\mathbb{R}^{2}\right)=0, \quad \mathrm{H}_{1}\left(\mathrm{~S}^{1} \times \mathrm{S}^{1}\right)=\mathbb{Z} \oplus \mathbb{Z}, \\
& \mathrm{H}_{2}\left(\mathbb{R}^{2}\right)=0, \quad \mathrm{H}_{2}\left(\mathrm{~S}^{1} \times \mathrm{S}^{1}\right)=\mathbb{Z} \quad \mathrm{e} \\
& \mathrm{H}_{j}\left(\mathbb{R}^{2}\right)=\mathrm{H}_{j}\left(\mathrm{~S}^{1} \times \mathrm{S}^{1}\right)=0, \quad j \geq 3 .
\end{aligned}
$$

Apenas em dimensão 2 notamos um discrepância de comportamento em relação a homotopia pois, ao invës de um isomorfismo, temos uma inclusão. Cabe, no entanto, observar que como o espaço $S^{1} \times S^{1}$ é nilpotente o teorema 5.3.4. do pröximo parägrafo nos permitirá explicar a forma pela qual esses grupos de homologia se relacionam. 
3) $\mathrm{S}^{\mathrm{n}} \longrightarrow \mathbb{R} \mathrm{P}^{\mathrm{n}}, \mathrm{n} \geq 1$.

Distinguiremos dois casos: a) n impar e b) n par.

a) $\mathrm{n}=2 \mathrm{k}+1$.

Também aqui o teorema 5.3.4. nos terả algo a dizer, pois, para valores impares de $n, \mathbb{R P}^{\mathrm{n}}$ è nilpotente.

Indicamos abaixo os grupos de homologia da base e do recobrimento para que o leitor os compare.

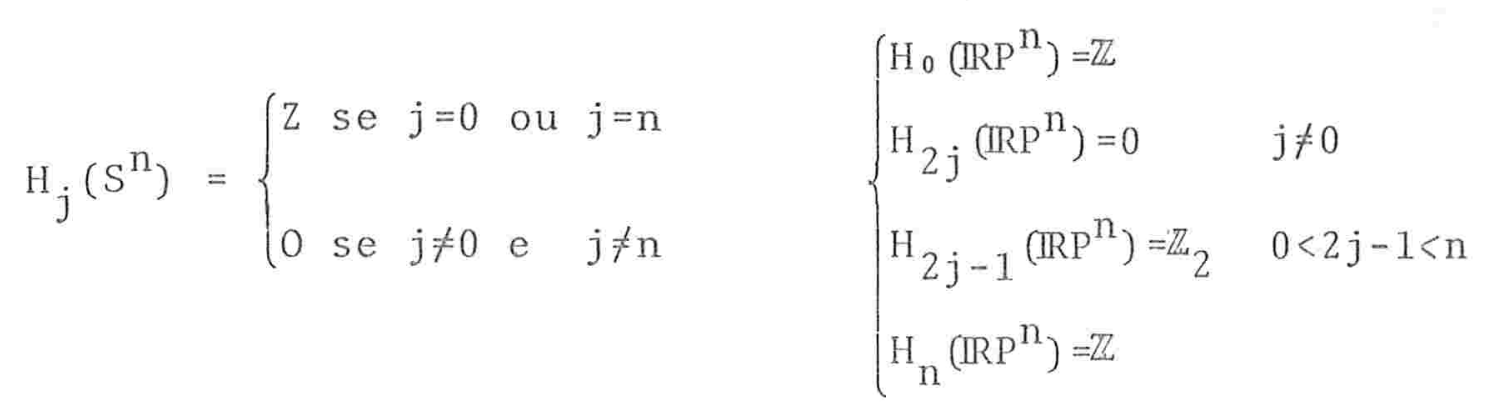
b) $\mathrm{n}=2 \mathrm{k}$.

Os grupos de homologia são os fornecidos pela tabela acima com uma ünica alteração: $H_{n}\left(\mathbb{R} P^{n}\right)=0$.

Por $n$ ser par, $\mathbb{R P}^{\mathrm{n}}$ não é nilpotente (confronte 3.3.3.) e, portanto, os resultados do pröximo parägrafo não são aplicäveis (confronte 5.3 .7$. ).

Estamos, como os exemplos acima sugerem, diante de uma situação bem mais delicada do que aquela com a qual. nos defrontamos no caso da homotopia.

o instrumento algébrico que nos permite relacionar a 
homologia de um espaço com a de seu recobrimento ê una sequência espectral introduzida por H. Cartan (confronte $[\mathrm{C}, \mathrm{S}])$ e que pode ser encarada como a sequência espectral de uma fibração associada ao recobrimento. Mais precisamente, seja $\mathrm{X} \longrightarrow \mathrm{X}$ um recobrimento regular cujo gru po de transformações indicamos por $\pi$. A esse recobrimento associamos a fibração $\bar{X} \longrightarrow X \longrightarrow K(\pi, 1)$ e, a essa fibra ção, sua sequência espectral. Essa sequência espectral $\vec{e}$ conhecida como sequência espectral do recobrimento $\bar{X} \rightarrow X$. Convém observar que, como $K(\pi, 1)$ não è simplesmente cone xo, precisamos trabalhar com coeficientes locais.

Um outro ponto para o qual chamamos a atenção do leitor é que interpretamos os grupos de homologia do espaço $K(\pi, 1)$ como sendo os grupos de homologia do grupo $\pi$. Em virtude disso, escrevemos $E_{p, q}^{2}=H_{p}\left(\pi, H_{q} \bar{X}\right)$ ao invës de $E^{2} p, q=H_{p}\left(K(\pi, 1), H_{q} \bar{X}\right)$

Finalmente, vale acrescentar que nem sempre $\vec{e}$ simples trabalhar com uma sequência espectral. Por vezes, os câlcu los se tornam penosos e, em consequência, menos efetivos. No entanto, na categoria dos espaços nilpotentes a situação, como nos foi possível mostrar nesse trabalho, simplifica-se bastante. Esse, porêm, è o objeto do próximo parägra fo. 
§3. RECOBRIMENTOS DE ESPAÇOS NILPOTENTES: HOMOLOGIA MOD C.

A partir de agora, focalizaremos nossa atenção nos espaços nilpotentes. Nosso objetivo, como já foi anunciado no parägrafo anterior, $\vec{e}$, utilizando o conceito de classe de Serre de grupos nilpotentes, relacionar a homologia de um espaço (nilpotente) com a homologia de seu recobrimento.

Inicialmente estudamos algumas propriedades dos recobrimentos de espaços nilpotentes que utilizaremos no decorrer do capítulo.

5.3.0. PROPOSICAO Se $X$ è un espaço nilpotente e $\bar{X} \longrightarrow X$ um recobrimento qualquer de $X$ então $\bar{X} \vec{e}$ nilpotente.

\section{DEMONSTRAÇÃO}

Como $\pi_{1} \bar{x} \vec{e}$ nilpotente (pois $\vec{e}$ isomorfo a um subgrupo do grupo nilpotente $\pi_{1} X$ ) precisamos apenas mostrar que $\pi_{1} \vec{X}$ opera nilpotentemente em $\pi_{n} \bar{X}(n \geq 2)$. E claro que, para isso, basta verificar que a ação (induzida pelo isomor fismo $\pi_{n} \bar{X} \stackrel{p *}{\rightarrow} \pi_{n} X, n \geq 2$ ) de $\pi_{1} \bar{X}$ em $\pi_{n} X \vec{e}$ nilpotente.

Um exame do diagrama

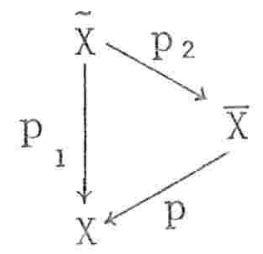


onde $\tilde{\mathrm{X}} \overrightarrow{\mathrm{e}}$ recobrimento universal de $\mathrm{X}$ e de $\overrightarrow{\mathrm{X}}$, 1evando em conta sua comutatividade e a unicidade dos levantamentos, nos mostra que a açäo de $\pi_{1} \bar{X}$ em $\pi_{n} X$ é a restrição da ação de $\pi_{1} X$ em $\pi_{n} X$ que é, por hípótese, nilpotente.

Como a restrição de uma ação nilpotente $\vec{e}$ uma ação nilpotente (confronte 3.1.4.) temos a tese.

Examinaremos agora o correspondente topológico do fato de todo subgrupo de um grupo nilpotente ser subnomal.

5.3.1. PROPOSICAO Seja $\bar{X} \stackrel{P}{\longrightarrow} X$ um recobrimento qualquer do espaço nilpotente $x$. Existe uma sequência $x_{k} \stackrel{p_{k}}{\rightarrow} x_{k-1}$, $k=1, \ldots, n$, de recobrimentos regulares de tal forma que

$$
\widetilde{x}=x_{n} \stackrel{p_{n}}{\rightarrow} x_{n-1} \longrightarrow x_{1} \stackrel{p_{1}}{\longrightarrow} x_{0}=x
$$

com $\mathrm{p}_{10} \mathrm{p}_{2} \quad \ldots \mathrm{p}_{\mathrm{n}}=\mathrm{p}$.

DEMONSTRACAO

Consideremos uma sẻrie subnormal

$$
\pi_{1} \bar{X}=G_{n} \Delta G_{n-1} \cdot \cdots G_{1} \Delta G_{0}=\pi_{1} X
$$

de subgrupos de $\pi_{1} X$ Iigando $\pi_{1} \bar{X}$ a $\pi_{1} X$.

0 teorema 5.1 .2 . nos permite construir un recobrimen to $X_{1} \stackrel{p_{1}}{\rightarrow} X$ do espaço $X$ tal que $\pi_{1} X_{1}=G_{1}$ e o homomorfismo $G_{1} \stackrel{\mathrm{P}_{1} *}{\rightarrow} \mathrm{G}_{0}=\pi_{1} X \overrightarrow{\mathrm{e}}$ a inclusäo. E claro, então, que um argumento de indução nos fornece a existência de reco 
brimentos

$$
\begin{aligned}
& x_{k} \stackrel{p_{k}}{\rightarrow} x_{k-1} \\
& \pi_{1} x_{k}=G_{k}, \quad \pi_{1} X_{k-1}=G_{k-1} \quad e
\end{aligned}
$$

o homomorfismo $G_{k} \stackrel{\rho_{k}^{*}}{\rightarrow} G_{k-1}$ e a inclusão do subgrupo normal $\mathrm{G}_{\mathrm{k}}$ em $\mathrm{G}_{\mathrm{k}-1}$.

Nessas condições

$$
\mathrm{x}_{\mathrm{n}} \longrightarrow \ldots \rightarrow \mathrm{x}_{0}=\mathrm{X}
$$

é um recobrimento no qual $\pi_{1} X_{n} \rightarrow \pi_{1} X \vec{e}$ a inclusão de $G_{n}$ em $\pi_{1} X$. Alêm disso, em virtude da unicidade estabelecida pelo teorema 5.1.2. ele é, (a menos de um isomorfismo canônico) o recobrimento $\bar{x} \stackrel{p}{\rightarrow} x$.

Consideremos agora uma classe de serre $C$ de grupos nil potentes:

5.3.2. PROPOSICAO Se supusermos que o recobrimento $\bar{X} \stackrel{p}{\rightarrow} X$ considerado na proposição 5.3.1. acima $\vec{e}$ tal que $\pi_{1} \vec{X} \longrightarrow \pi_{1} X$ é uma C-sobrejeção, a sequência de recobrimentos regulares

$$
\bar{x}=x_{n} \stackrel{p_{n}}{\longrightarrow} x_{n-1} \longrightarrow \ldots \rightarrow x_{1} \stackrel{p_{3}}{\rightarrow} x_{0}=x
$$

pode ser construida de forma que

$$
\pi_{1} x_{k-1} \int_{p_{k}^{*} x_{1} x_{k}} \in c \quad k=1, \ldots, n .
$$


DEMONSTRAÇÃO

Como $\pi_{1} \bar{X} \longrightarrow \pi_{1} X$ è uma $C$-sobrejeção existe (confronte 3.2.4.) uma sërie subnormal de subgrupos

$$
\vec{X}=G_{n} \Delta G_{n-1} \quad \cdots \quad G_{1} \Delta G_{0}=\pi_{1} X
$$

tal que $\mathrm{G}_{\mathrm{k}-1 / \mathrm{G}_{\mathrm{k}}} \in \mathrm{c}$.

constroi-se então os recobrimentos como em 5.3.1.

Nosso pröximo resultado ê um lema de caräter técnico e que será importante para a demonstração do teorema 5.3.4. Sua demonstração pressupõe alguma familiaridade com [H]. Para facilidade do leitor incluimos, no capítulo III, os enunciados das proposições explicitamente mencionadas no decorrer da demonstração do Iema.

5.3.3. LEMA Seja $X$ um espaço nilpotente e $\pi$ o grupo de transformações de recobrimento de um recobrimento regular $\bar{X} \stackrel{p}{\rightarrow} X$ de $X$. A ação de $\pi$ em $H_{q} \bar{X}$ è nilpotente qualquer que seja o inteiro positivo q.

DEMONSTRAÇAO

Para simplificar a notaçäo escreveremos $\pi_{1}=\pi_{1} X, \bar{\pi}_{1}=\pi_{1} \bar{X}_{0}$ Consideremos a fibração $\tilde{\mathrm{X}} \longrightarrow \mathrm{X} \longrightarrow \mathrm{K}\left(\bar{\pi}_{1}, 1\right) \mathrm{em} \mathrm{cu-}$ jos grupos de homologia o grupo $\pi_{1}$ opera de forma obvia. $\tilde{x}$ 
representa aqui o recobrimento universal de $x$. Observemos, de início, que essa é uma fibração nilpotente com fibra nilpotente e, portanto, pelo teorema 3.3.6. ela $\vec{e}$ também quase-nilpotente. Por outro 1ado, o teorema 3.3.2. nos assegura que a ação de $\pi_{1}$ na homologia da fibra $\tilde{x} \vec{e}$ nilpotente. Sendo $\pi_{1}$ um grupo nilpotente, sua ação (por conjugação) em $\bar{\pi}_{1}$ è nilpotente [H]. A proposição 3.1.6. nos diz então que a ação $\pi_{1}$ na homologia de $K\left(\bar{\pi}_{1}, 1\right)$ tam bêm ê nilpotente. Invocamos agora o teorema 3.3.7. e con cluimos que $\pi_{1}$ age nilpotentemente na homologia de $\bar{X}$. Pa ra termos a tese, basta observar que a ação de $\pi_{1}$ na homologia de $\bar{X}$ ê induzida da ação de $\pi$ através do epimor-fismo $\varepsilon$ que figura na sequência exata

$$
\bar{\pi}_{1} \longrightarrow \pi_{1} \longrightarrow \pi
$$

Chegamos agora ao principal resultado deste capitulo.

Indicaremos por C uma classe de Serre pröpria e acíclica de grupos nilpotentes.

5.3.4. TEOREMA Se $\overline{\mathrm{X}} \stackrel{\mathrm{p}}{\rightarrow} \mathrm{x}$ é um recobrimento regular de um espaço nilpotente $X$ e se ou $x$ é de tipo finito ou $c \vec{e}$ completa então as seguintes condições são equivalentes:

i) $\pi \in C$, onde $\pi \vec{e}$ o grupo de transformações de reco brimento do recobrimento $\bar{x} \stackrel{p}{\longrightarrow} x$. 
ii) O homorfismo induzido na homologia $\mathrm{H}_{\mathrm{q}} \mathrm{X} \longrightarrow \mathrm{H}_{\mathrm{q}} \mathrm{X}$ pe la projeção do recobrimento $\vec{e}$ uma $c$-bijeção para todo in teiro $q$.

DEMONSTRAÇÃO

1) ii) $\Rightarrow$ i)

Como $\vec{X} \stackrel{p}{\rightarrow} X \vec{e}$ um recobrimento regular, $\pi \vec{e}$ isomorfo ao quociente $\pi_{1} \mathrm{x} / \int_{p_{*} \pi_{1} \bar{x}}$ e portanto o que temos que mos$\operatorname{trar} \vec{e}$ que $\pi_{1} X \int_{p * \pi_{1} \bar{X}} \in C$. Utilizando-se a propriedade $u-$ niversal que caracteriza o funtor abelianização e o fato de ser ele um funtor exato a direita verifica-se facilmente que o abelianizado do grupo $\pi_{1} \mathrm{X} / \mathrm{p}_{\mathrm{*} \pi_{1} \overline{\mathrm{X}}} \overrightarrow{\mathrm{e}} \mathrm{o}$ conucleo do homomorfismo $H_{1} \bar{X} \longrightarrow H_{1} X$ que, por hipótese, pertence a $C$.

o teorema 3.2.1. do capitulo III, que diz que um gru po pertence a $C$ se e somente se o seu abelianizado pertencer a $C_{1}$ nos garante então que $\pi_{1} X /_{p * \pi_{1} \bar{X}} \in C$.

2) i) $\Rightarrow$ ii)

Como, pelo lema 5.3.3., a ação de $\pi$ em $H_{q} \vec{X} \vec{e}$ nilpo- 
tente o corolärio 4.1.4. do capítulo IV nos assegura que $\mathrm{H}_{\mathrm{q}} \overline{\mathrm{X}} \longrightarrow\left(\mathrm{H}_{\mathrm{q}} \overline{\mathrm{X}}\right)_{\pi} \overrightarrow{\mathrm{e}}$ uma $\mathrm{C}-\mathrm{b}$ ijeção.

Utilizaremos a sequência espectral do recobrimento $\bar{X} \longrightarrow X$ para provar que existe uma $C$-bijeção entre $\left(H_{q} \bar{X}\right)_{\pi}$ e $H_{q}(X)$. E claro que, havendo estabelecido esta $C$-bijeção, te remos demonstrado o teorema.

Temos

$$
\begin{aligned}
& E_{0, q}^{2}=H_{0}\left(\pi, H_{q} \bar{X}\right)=\left(H_{q} \bar{X}\right) \pi
\end{aligned}
$$

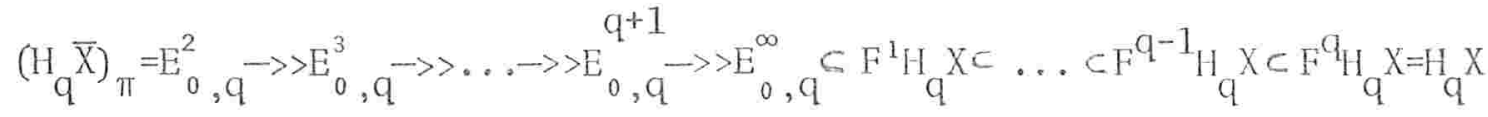

$$
\begin{aligned}
& \uparrow d^{2} \uparrow d^{3} \\
& \mathrm{~d}^{\mathrm{q}+1} \\
& \downarrow \\
& \mathrm{E}_{2, \mathrm{q}-1} \mathrm{E}_{3, \mathrm{q}-2} \\
& E_{q+1}^{q+1}, 0 \\
& E_{1, q-1}^{\infty} \\
& \mathrm{E}_{\mathrm{q}-1,1}^{\infty}
\end{aligned}
$$

o diagrama acima representa uma parte - a relevante para o nosso argumento - da sequência espectral do recobrimento $\bar{X} \longrightarrow X \cdot F^{1} H_{q} X, \ldots, F^{q_{H}} X$ são os termos da filtração natural de $\mathrm{H}_{\mathrm{q}} \mathrm{X}$.

Como a ação de $\pi$ em $\mathrm{H}_{\mathrm{q}} \overline{\mathrm{X}}$ ê nilpotente o teorema 4.1.1. do capitulo IV nos permite afirmar que $E_{j, q-j+1}^{2} \in C$, $j=2, \ldots, q+1$. E c1aro então que $E_{j, q-j+1}^{j} \in c \quad$ para $j=2, \ldots, q+1$ o que nos permite concluir ser o homomorfismo

$$
E_{0}^{2}, q_{0} \rightarrow E^{3}, q^{->>} \rightarrow>E_{0, q}^{q+1} \rightarrow>E_{0, q}^{\infty}
$$


uma C-bijeção.

Mostraremos agora que as inclusões

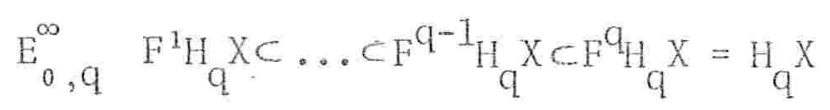

são c-sobrejeções.

Para isso basta verificar que os respectivos conücleos $E_{1, q-1}^{\infty}, E_{2, q-2}^{\infty}, \ldots, E_{q-1,1}^{\infty}$ estão em $c$.

Novamente estamos em condições de aplicax o teorema 4.1.1. do capitulo $I V$ e dizer que $E_{j, q-j}^{2} \in C, \quad j=1, \ldots, q-1$; consequentemente $E_{j, q-j}^{\infty} \in C, j=1 \ldots, q-1$ e portanto as inclusões acima são $C$-sobrejeções.

Mantendo as mesmas notaçöes e hipóteses de 5.3.4. podemos enunciar um corolário (na realidade um caso particu lar do teorema 5.3 .4$.$) .$

5.3.5. COROLARIO Se $\tilde{\mathrm{x}} \longrightarrow \mathrm{x}$ eे o recobrimento universal

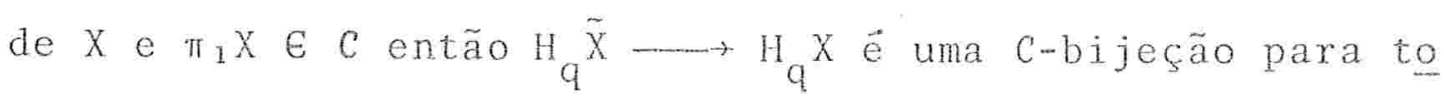
do inteiro $q$.

DEMONSTRAÇAO

O grupo de transformações de recobrimento do recobrimento universal $\tilde{X} \longrightarrow x \vec{e}$ isomorfo a $\pi_{1} X$.

A propriedade de subnormalidade dos subgrupos de grupos nilpotentes nos permite estender (na direção i) $\Rightarrow$ ii)) 0 
teorema 5.3 .4 .

5.3.6. COROLARIO Seja $\bar{X} \longrightarrow x$ um recobrimento (não necessariamente regular) do espaço nilpotente $X$. Se o homo morfismo $\pi_{1} \bar{X} \longrightarrow \pi_{1} X$ e uma $C$-sobrejeção então os homomor fismos $\mathrm{H}_{\mathrm{q}} \overline{\mathrm{X}} \longrightarrow \mathrm{H}_{\mathrm{q}} \mathrm{X}$ são $\mathrm{C}$-bijeções para todo inteiro $\mathrm{q}$.

DEMONSTRAÇÃO

"Fatora-se" como em 5.3.2. o recobrimento $\vec{X} \longrightarrow \mathrm{X}$ nu ma sequência

$$
\overline{\mathrm{x}} \simeq \mathrm{x}_{\mathrm{n}} \stackrel{\mathrm{p}_{\mathrm{n}}}{\rightarrow} \mathrm{x}_{\mathrm{n}-1} \longrightarrow \ldots \mathrm{x}_{1} \stackrel{\mathrm{p}_{1}}{\longrightarrow} \rightarrow \mathrm{x}_{0}=\mathrm{x}
$$

de recobrimentos regulares de tal forma que $p_{1} \circ p_{2} \ldots p_{n}=p$

$$
\pi_{1} x_{j-1} / p_{j * \pi_{1} x_{j}} \in \quad c .
$$

Aplica-se então o teorema 5.3.4. a cada um dos recobrimentos $x_{j} \stackrel{p_{j}}{\rightarrow} x_{j-1}, j=1, \ldots, n$ obtendo-se as $c-b i j \underline{e}$ ções $H_{q}\left(X_{j}\right) \longrightarrow H_{q}\left(X_{j-1}\right)$.

Compondo-se essas C-bijeções temos a tese.

\subsubsection{COMENTARIOS}

1) Verifica-se sem dificuldade que o corolârio 5.3.5. permanece välido se supusermos apenas que:

i) $\pi_{1} X$ opera nilpotentemente na homologia de $X$.

ii) $H_{i} \pi \in C, i \geqslant 1$ onde $\pi=\pi_{1} X$. 
Espaços satisfazendo a condição i) estão sendo estuda dos com o nome de homologicamente nilpotentes.

Essa denominação é devida a Dror e Dwyer.

2) 0 teorema 5.3 .4 e seus corolärios permanecem vâli dos se considerarmos a homologia com coeficientes num gru po abeliano A (finitamente gerado quando a classe $C$ não for completa). Verifica-se esse fato considerando-se o diagrama comutativo

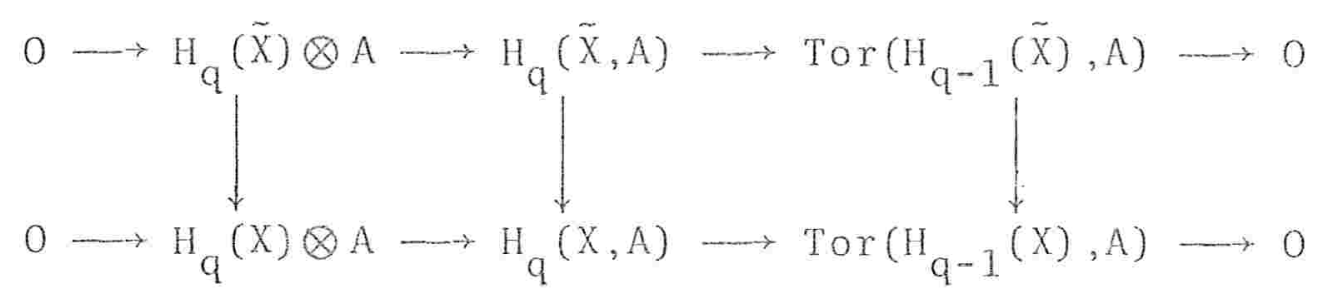

cujas linhas são dadas pelo teorema dos coeficientes universais. Como as flechas verticais das extremidades são, pela proposição 4.1.6., C-bijeções, a flecha vertical do centro é tambêm uma C-bijeção.

3) O leitor näo terá deixado de perceber que quando a classe c é formada apenas por grupos triviais (grupos com um único elemento) o teorema 5.3.4. perde toda sua força pois afirma apenas que a aplicação identidade $\mathrm{x} \rightarrow \mathrm{x}$ in duz isomorfismos na homologia. Esse é o primeiro exemplo desse tipo que conhecenos, isto $\vec{e}$, de um teorema que envolvendo a noção de classe de grupos na sua formulação tor na-se genuinamente trivial quando consideramos a classe 
trivial.

\subsubsection{CONTRA-EXEMPLO}

Examinaremos agora um exemplo que nos mostrarä que al guma hipótese de nilpotência é necessäria para a validade do teorema 5.3.4. e seus corolärios. Mais precisamente, da remos um contra-exemplo ao corolärio 5.3.5. no caso da hi pötese da nilpotência do espaço não estar verificada.

Consideremos o recobrimento universal $\mathrm{S}^{2} \stackrel{\mathrm{P}}{\rightarrow} \operatorname{IRP}^{2}$ de $\mathbb{R P P}^{2}$ e seja $C$ a classe dos grupos nilpotentes de torção. Deixamos aos cuidados do leitor a verificação de que essa classe é completa e acíclica. E claro que $\mathbb{I R P}^{2}$ é um espaço de tipo finito. Por outro lado, jā vimos no capítulo III (confronte 3.3 .3 ) que $\mathbb{R P}^{2}$ não é um espaço nilpotente. (Na realidade, mostramos que $\mathbb{R P}^{2}$ não ê nem mesmo homologicamente nilpotente)

Como $\pi_{1}\left(\mathbb{R P}^{2}\right)=\mathbb{Z} / 2 \mathbb{Z} \in C$, a ünica hipótese do corolário 5.3.5. que não está satisfeita é a de nilpotência do espa Ç० $\mathbb{R P P}^{2}$.

Finalmente, obtemos o contra-exemplo examinando o homomorfismo

$$
\mathrm{H}_{2}\left(\mathrm{~S}^{2}\right) \stackrel{\mathrm{P} *}{\rightarrow} \mathrm{H}_{2}\left(\mathrm{IRP}^{2}\right)
$$

Sendo $\mathrm{H}_{2}\left(\mathrm{~S}^{2}\right)=\mathbb{Z}$ e $\mathrm{H}_{2}\left(\mathbb{R} P^{2}\right)=0$

vemos que ker $\mathrm{p} *=\mathbb{Z} \mathbb{C} \mathcal{C}$ e, portanto, $\mathrm{p} *$ não $\overrightarrow{\mathrm{e}}$ uma $\mathcal{C}$-bije ção. 


\section{\$4. CASO RELATIVO}

Mostraremos agora una maneira simples de transportar para o caso relativo o corolärio 5.3.5. Esse resultado nos permitirä demonstrar, no próximo capîtulo, uma forma rela tiva do teorema de Hurewicz. Devemos no entanto ressaltar que esse não $\vec{e}$, aparentemente, o melhor resultado nessa direção. Acreditamos poder demonstrar, por métodos simila res aos aqui desenvolvidos, um resultado substancialmente mais forte.

5.4.0. Seja $(X, A)$ um par de espaços. Indicaremos por $\tilde{X} \longrightarrow X$ e $\tilde{A} \longrightarrow A$ os recobrimentos universais de $X$ e A. E claro que podemos "levantar" a inclusão $A \stackrel{i}{\rightarrow} x$ obtendo uma aplicação continua $\tilde{A} \stackrel{I}{\longrightarrow} \tilde{X}$ e portanto, a menos de uma equivalência de homotopia, teremos uma inclusão de $\tilde{A}$ em $\tilde{X}$. Em virtude disso, estaremos, nas considerações abai xo, num abuso usual de linguagem, supondo $\tilde{A}$ um subespaço de $\tilde{X}$ 。

Diremos que $(X, A)$ é um par nilpotente se $X$ e A são es paços nilpotentes. Se $\mathrm{X}$ e A forem de tipo finito diremos que o par $(X, A) \vec{e}$ de tipo finito.

Como de costume, $\mathrm{C}$ indicara una classe Serre pröpria e acíclica de grupos nilpotentes. 
5.4.1. PROPOSICAO Seja (X,A) um par nilpotente e suponhamos que $\pi_{1} A$ e $\pi_{1} X$ estão em $C$. Se o par $(X, A)$ è de tipo finito ou a classe C completa, o homomorfismo. $\mathrm{H}_{\mathrm{q}}(\tilde{X}, \tilde{A}) \longrightarrow \mathrm{H}_{\mathrm{q}}(X, A) \bar{e}$, para todo inteiro $\mathrm{q}$, uma $c$-bije Ção.

DEMONSTRAÇAOO

A projeção $(\tilde{X}, \tilde{A}) \longrightarrow(X, A)$ induz um homomorfismo en tre as sequências exatas longas de homologia desses pares formando o diagrama comutativo

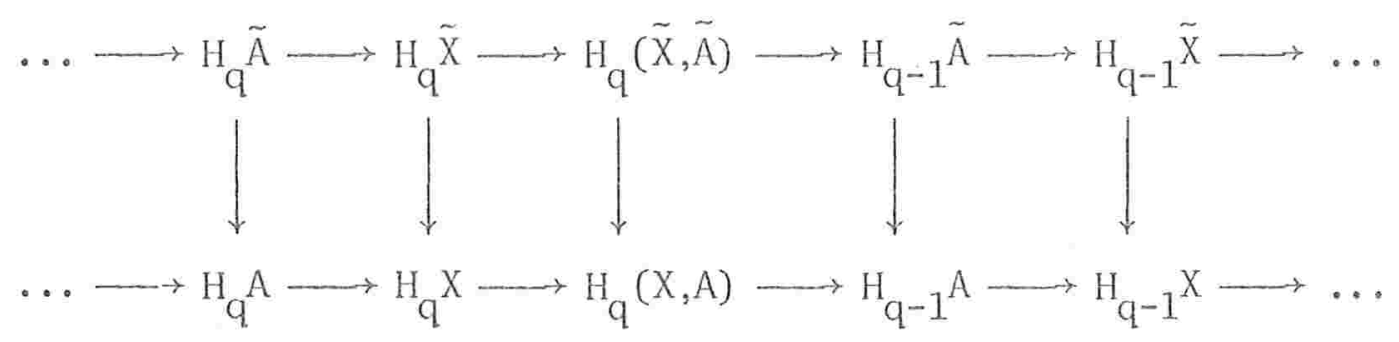

Estando as hipóteses do corolärio 5.3.5. verificadas, concluimos que as quatro flechas verticais laterais são C-bijeções. o lema dos cinco möduclo c (teorema 3.2.6.) nos garante então que o homomorfismo

$$
\mathrm{H}_{\mathrm{q}}(\tilde{\mathrm{X}}, \tilde{\mathrm{A}}) \longrightarrow \mathrm{H}_{\mathrm{q}}(\mathrm{X}, \mathrm{A})
$$

è uma C-bijeção 


\section{\$. COHOMOLOGIA}

Como jă dissemos reiteradas vezes, não $\vec{e}$ nossa intenção, neste trabalho, tratar em condições de igualdade, ho mologia e cohomologia. Na realidade, nem teriamos, a esta altura, condiçöes de fazê-1o pois não desenvolvemos suficientemente, no que se refere à cohomologia, o material algêbrico do capîtulo IV.

Nös nos restringimos à seguinte questão:

Seja $X \stackrel{f}{\longrightarrow} Y$ uma função continua tal que, para todo inteiro $\mathrm{q}$, o homomorfismo $\mathrm{H}_{\mathrm{q}} \mathrm{X} \longrightarrow \mathrm{H}_{\mathrm{q}} \mathrm{Y}$ ë uma $\mathrm{c}$-bijeção. Se-rä que nessas condiçōes $f$ induz c-bijeçöes tambëm entre os grupos de homologia? A proposição abaixo dả uma condição suficiente para que isso aconteça.

5.5.0. PROPOSICAO Seja B un grupo abeliano finitamente gerado $e X$ e $Y$ espaços nilpotentes de tipo finito. Se a função continua $X \stackrel{f}{\longrightarrow} Y$ for tal que $\mathrm{H}_{\mathrm{q}} \mathrm{X} \stackrel{\mathrm{f}_{*}}{\longrightarrow} \mathrm{H}_{\mathrm{q}} \mathrm{Y}$ é uma C-bijeção para $q \geq 0$, então $H^{q}(Y, B) \longrightarrow H^{q}(X, B)$ tambëm serä uma $\mathrm{C}$-bijeção para todo inteiro $\mathrm{q}$.

DEMONSTRAÇATO

Consideremos o seguinte diagrama comutativo 


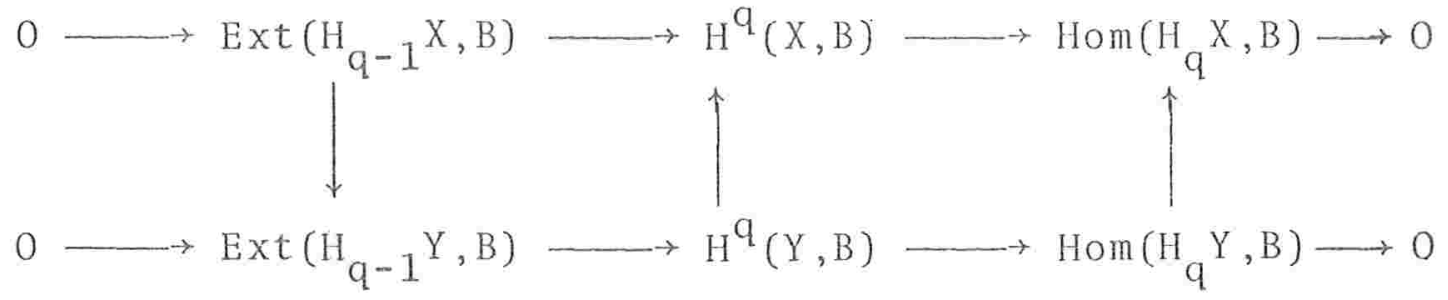

construido a partir do teorema dos coeficientes universais para a cohomologia. Como as flechas verticais laterais são, em virtude da proposição 4.2 .2$. , C-bijeçöes concluimos, utilizando a proposição 3.2.6., que $\mathrm{f} *$ é uma C-bijeção.

A proposição acima, o corolário 5.3.6. e o fato de todo recobrimento de um espaço de tipo finito ser também um espaço de tipo finito nos permite enunciar a seguinte.

5.5.1. PROPOSICAO Seja B um grupo finitamente gerado, $X$ um espaço nilpotente de tipo finito e $\bar{X} \longrightarrow X$ um recobrimento (não necessariamente regular) de $\mathrm{X}$. Se o homomorfismo $\pi_{1} \bar{X} \longrightarrow \pi_{1} X$ for uma $C$-sobrejeção, os homomorfismos $H^{q}(X, B) \longrightarrow H^{q}(\bar{X}, B)$ serão $C$-bijeções para todo inteiro $q$. 

CAPITULO VI

APLICAÇÕES. O TEOREMA DE HUREWICZ MODDULO

C E A SEQUENCIA EXATA DE J.H.C.WHITEHEAD

§. INTRODUÇÃO

Encerramos o trabalho mostrando como utilizax o teorema 5.3 .4 e seus corolärios para estabelecer na categoria dos es paços nilpotentes alguns resultados que na teoria de Homoto pia clâssica exigiam, para sua validade, a hipötese de que os espaços envolvidos fossem simplesmente conexos.

$\mathrm{Na}$ realidade, essas aplicações do teorema 5.3 .4 ilustram uma nova maneira de desenvolver o programa de generalizar,pa ra a categoria dos espaços nilpotentes, métodos que provaram ser de grande utilidade no estudo da teoria de homotopia dos espaços simplesmente conexos.

Especificamente, o que fizemos neste capitulo foidemons trar o teorema de Hurewicz módulo $C$ no caso absoluto e dar 
uma versão (que acreditamos possa ser melhorada) desse mesmo. teorema no caso relativo. Demonstramos também, no contexto das classes de Serre de grupos nilpotentes, uma propriedade relativa aos grupos $\Gamma_{i} X$ que comparecem na sequência exata de J.H.C.Whitehead que utilizamos no capitulo I pa ra provar o addendum ao teorema de Hurewicz clässico.

Como de hábito, indicaremos por c uma classe de Serre própria e aciclica de grupos nilpotentes. 


\section{$\S 1$. APLICACOES}

Ocuparemo-nos, em primeiro lugar, do caso absoluto, apresentando uma versão mais forte de um resultado obtido or $\underline{i}$ ginalmente por Hilton e Roitberg (teorema 5.1 de $[H, R]_{1}$. 6.1.0 TEOREMA. Seja $X$ um espaço nilpotente e $c$ uma classe de Serre pröpria e acïclica de grupos nilpotentes. Suponhamos que ou (a)X è de tipo finito ou (b)c è completa. Então as se guintes condições são equivalentes:

(i) $\pi_{i} X \in C \quad 1 \leq i<n$

(ii) $H_{i} X \in C \quad 1 \leq i<n$.

$\mathrm{Na}$ eventualidade de uma dessas (e portanto ambas) condi ções ser verdadeira podemos afirmar que

(iii) $\pi_{n} \mathrm{X} \longrightarrow \mathrm{H}_{\mathrm{n}} \mathrm{X} \ddot{\mathrm{e}}$ uma c-bijeção

(iv) $\pi_{n+1} X \longrightarrow H_{n+1} X$ e uma c-sobrejeção.

DEMONSTRACAO

Começaremos provando que as condições (i) e (ii) são equivalentes.

Observamos, de início, que

$$
\pi_{1} \mathrm{XEC} \Longleftrightarrow \mathrm{H}_{1} \in \mathrm{C}
$$

pois $H_{1} X$ è o abelianizado de $\pi_{1} X$.

Consideremos o recobrimento universal $\tilde{X} \rightarrow X$. Como $\tilde{X} e$ simplesmente conexo, o teorema 2.2 .0 nos diz que, se $1 \leq j<n$, 
vale a equivalência

$$
\pi_{j} \tilde{X} \in C \Longleftrightarrow H_{j} \tilde{X} \in C .
$$

Levando em conta que se $j \geq 2, \pi_{j} \tilde{X}=\pi_{j} X$, podemos garantir que para todo inteiro $j \geq 2$

$$
\pi_{j} X \in C \Longleftrightarrow H_{j} \tilde{X} \in C \text {. }
$$

Por outro lado o corolário 5.3 .5 nos assevera que para todo inteiro $j$ o homomorfismo

$$
H_{j} \tilde{X} \longrightarrow H_{j} X
$$

$\vec{e}$ uma $C$-bijeção e portanto

$$
\mathrm{H}_{j} \tilde{\mathrm{X}} \in \mathrm{C} \Longleftrightarrow \mathrm{H}_{j} \mathrm{X} \in \mathrm{C}
$$

As equivalências (1), (2) e (3) nos permitem concluir que se $j \geq 2$

$$
\pi_{j} X \in C \Longleftrightarrow H_{j} X \in C
$$

Como jả observamos que essa equivalência vale tambëm pa ra $j=1$ temos a tese, isto $\vec{e},(i) \Longleftrightarrow$ (ii).

Resta-nos demonstrar (iii) e (iv).

Consideraremos novamente o recobrimento universal $\tilde{\mathrm{X}} \longrightarrow x$ e manteremos as notaçöes utilizada acima.

Como $\tilde{x} \ddot{e}$ simplesmente conexo, os itens (iii) e (iv) do teorema 22.0 nos garantem que 


$$
\begin{aligned}
& \pi_{n} \tilde{\mathrm{X}} \longrightarrow \mathrm{H}_{\mathrm{n}} \tilde{\mathrm{X}} \overrightarrow{\mathrm{e}} \text { uma } c \text {-bijeção } \\
& { }_{n+1} \tilde{\mathrm{X}} \longrightarrow \mathrm{H}_{n+1} \tilde{\mathrm{X}} \ddot{\mathrm{e}} \text { uma } C \text {-sobrejeção. }
\end{aligned}
$$

Conclui-se a demonstração utilizando-se os isomorfismos, $\pi_{n} \tilde{X}=\pi_{n} X, \pi_{n+1} \tilde{X}=\pi_{n+1} X$ e fato de serem os homomorfismos

$$
\mathrm{H}_{j} \tilde{\mathrm{X}} \longrightarrow \mathrm{H}_{j} \mathrm{X}
$$

C-bijeções.

Convëm observar que o teorema 5.1 de $[H, R]_{1}$ não inclui a afirmação de que $\pi_{n+1} X \longrightarrow H_{n+1} X \vec{e}$ una $C$-sobrejeção e garante apenas que $\pi_{n}^{x} \mathrm{X} \rightarrow \mathrm{H}_{\mathrm{n}} \mathrm{X} \overrightarrow{\mathrm{e}}$ uma $\mathrm{C}$-bijeção. $\pi_{n}^{\prime} \mathrm{X}$ indica $a-$ qui, o grupo obtido pela fatoração da ação de $\pi_{1} X$ em $\pi_{n} X$.

Trataremos agora do caso relativo. Näo queremos enfatiză-1o muito pois, como jâ dissemos, acreditamos poder melho rar o resultado que apresentamos aqui.

6.1.1 TEOREMA. Seja $(X, A)$ um par nilpotente e suponhamosque $\pi_{1} A$ e $\pi_{1} X$ estão em $C$. Se o par $(X, A) \vec{e}$ do tipo finito ou a classe $C$ é completa então as seguintes condições são equiva lentes:

(i) $\pi_{i}(X, A) \in C \quad 1 \leq i \leq n-1$

(ii) $H_{i}(X, A) \in C \quad 1 \leq i \leq n-1$.

$\mathrm{Na}$ eventualidade de uma (e portanto ambas) dessas condi ções estar verificada podemos garantir que:

$$
\begin{aligned}
& \text { (iii) } \pi_{n}(X, A) \longrightarrow H_{n}(X, A) \text { è uma } C \text {-bijeção } \\
& \text { (iv) } \pi_{n+1}(X, A) \longrightarrow H_{n+1}(X, A) \text { è uma c-sobrejeção. }
\end{aligned}
$$


DEMONSTRAÇAO

Observemos inicialmente que, em virtude de

$$
\pi_{1} \mathrm{X} \longrightarrow>\pi_{1}(\mathrm{X}, \mathrm{A}) \text { e } \mathrm{H}_{1} \mathrm{X} \longrightarrow>\mathrm{H}_{1}(\mathrm{X}, \mathrm{A})
$$

serem epimorfismos e $\pi_{1} X$ e $\mathrm{H}_{1} \mathrm{X}$ estarem em $\mathrm{C}\left(\mathrm{H}_{1} \mathrm{X}\right.$ pertence a $C$ por ser o abelianizado de $\left.\pi_{1} X\right)$, os grupos $\pi_{1}(X, A)$ e $H_{I}(X, A)$ estão em $C$.

A seguir consideremos o diagrama comutativo

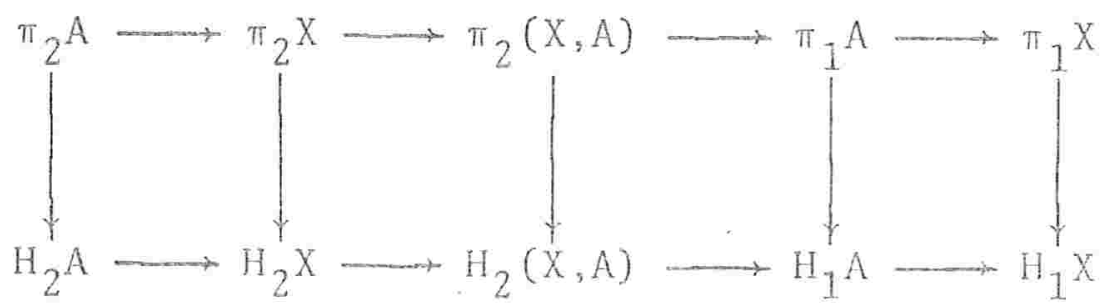

cujas Iinhas são exatas.

As duas flechas verticais da esquerda são, pelo teorema de Hurewicz absoluto (teorema 6.1.0) C-bijeções. As duas da direita são tambëm C-bijeções pois seus dominios e contradominios são grupos pertencentes a classe C. O lema dos cin co (teorema 3.2 .6$)$ nos garante então que $\pi_{2}(X, A) \rightarrow H_{2}(X, A)$ $\vec{e}$ uma $C$-bijeção.

As considerações acima mostram que para $j=1,2$ a equiva lência i) $\Longleftrightarrow$ ii) estâ automaticamente verificada.

Introduzindo-se o recobrimento universal $(\tilde{X}, \tilde{A}) \rightarrow(X, A)$ do $\operatorname{par}(X, A)$, - a observação que fizemos em 5.4.0 vale tam- 
bẻm aqui - utilizando-se o teorema 2.2.1 e a proposição 5.4.1 obtemos, argumentando da mesma forma que no caso abso 1 uto (teorema $(6.1 .0)$, a tese.

E bem conhecido que do teorema de Hurewicz relativo podemos deduzir o teorema de Whitehead. Näo exploraremos este fato aqui por não acreditamos, como jä dissemos reiteradas vezes, estar de posse da melhor forma do teorema de Hurewicz relativo. Queremos, no entanto, chamar a atenção do leitor para o fato de existirem versões do teorema de White head para o caso nilpotente. Confronte-se, por exemplo, $[H, R]_{1},[H, R]_{2}$ e $[D]$.

Esses resultados foram obtidos por outras vias uma vez que não se dispunha do teorema de Hurewicz relativo no caso nilpotente.

Em particular Hilton e Roitberg obtiveram uma forma do teorema de whitehead (confronte corolärio 3.4 de $[H, R]_{2}$ ) que admite como consequência imediata o corolärio 5.3 .5 do capï tulo antexior. Devemos, no entanto, observar que os métodos por eles empregados são extremamente elaborados enquanto os nossos são de natureza elementar.

Concluiremos o capitulo provando uma propriedade dos gru pos $\Gamma_{i} X$ que figuram na sequência exata de J.C.H. Whitehead. As definições e notações relevantes foram introduzidas em 1.2 .0 . 
6.1.2 TEOREMA. Seja $x$ um espaço nilpotente e $C$ uma classe de Serre própria e acíclica de grupos nilpotentes. Suponha que $X \vec{e}$ de tipo finito ou que $c \vec{e}$ completa. Nessas condições, po demos afirmar que se $\pi_{i} X \in C, 1 \leq i \leq n-1$, então $\Gamma_{i} X \in C, 1 \leq i \leq n$. DEMONSTRACATO

Seja $\tilde{X} \longrightarrow X$ o recobrimento universal de $X$ e consideramos a sequência exata

$$
\cdots \pi_{j+1} \tilde{x} \stackrel{h_{j+1}}{\longrightarrow} H_{j+1} \tilde{x} \stackrel{\gamma_{n+1}}{\longrightarrow} T_{j} \tilde{x} \stackrel{\lambda_{j}}{\longrightarrow} \pi_{j} \tilde{x} \stackrel{h_{j}}{\longrightarrow} H_{j} \tilde{x} \stackrel{\gamma_{j}}{\longrightarrow} \Gamma_{j-1} \tilde{x} \longrightarrow \cdots
$$

introduzida em 1.2.0.

Em virtude do isomorfismo $\pi_{j} \tilde{X}=\pi_{j} X, j \geq 2$, podemos reescrever essa sequência como

$$
\cdots \longrightarrow \pi_{j+1} X \stackrel{h}{\longrightarrow}{ }_{j+1}{ }_{j} \tilde{X} \stackrel{\gamma+1}{\longrightarrow} \Gamma_{j} X \stackrel{\lambda_{j}}{\longrightarrow} \pi_{j} x \stackrel{h}{\longrightarrow} H_{j} \tilde{X} \stackrel{\gamma_{j}}{\longrightarrow} \Gamma_{j-1} X \longrightarrow \cdots
$$

Se $j \leq n-1$, o teorema 6.1 .0 nos garante que $H_{j} X \in C$ e, por tanto, pelo corolärio 5.3.5, temos, que $H_{j} \tilde{X} \in C$. Em vista dis so, aplicando-se a proposição 3.2.10 à sequência exata acima conclui-se imediatamente que, para $j \leq n-2, \Gamma_{j} X \in C$

Provaremos agora que $\Gamma_{n-1} X \in C$.

Os isomorfismos e igualdades abaixo

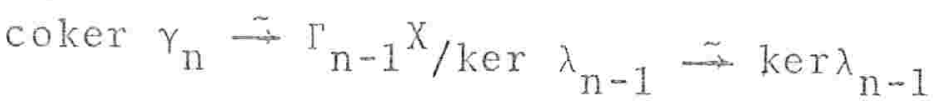

$$
\begin{aligned}
& \text { ker } \lambda_{n-1}=\operatorname{Im} \gamma_{n} \stackrel{\sim}{\rightarrow} \operatorname{coker} h_{n}
\end{aligned}
$$


decorrem facilmente da exatidão da sequência

$$
\pi_{n} x \stackrel{h_{n}}{\longrightarrow} H_{n} x \stackrel{\gamma_{n}}{\longrightarrow} \Gamma_{n-1} x \stackrel{\lambda_{n-1}}{\longrightarrow} \pi_{n-1} x
$$

Alëm disso, Im $\lambda_{n-1}$ pertence a $C$ por ser subgrupo do gru po $\pi_{n-1} X$ que, por hipótese, estä em $C$. Invocando (1) deduzi mos que $\Gamma_{n-1} X /$ ker $\lambda_{n-1}$ pertence a $c$. Como ker $\lambda_{n-1}$ estä em $c$ (por (2), $\operatorname{ker} \lambda_{n-1} \stackrel{\sim}{\rightarrow} \operatorname{coker} h_{n}$ e coker $h_{n}$ estä em $C$ pois, pelo item iii) do teorema 6.1.0, $h_{n} \vec{e}$ uma $C$-bijeção). Concluímos, utilizando a proposição 3.2 .8 que $\Gamma_{n-1} X$ pertence a c.

A prova de que $\Gamma_{n} X$ esta em C utiliza os isomorfismos e igualdades abaixo

$$
\begin{aligned}
\Gamma_{n} X / \operatorname{Im} \gamma_{n+1} & \rightarrow \operatorname{Im} \lambda_{n}=\operatorname{ker} h_{n} \\
\operatorname{Im} \gamma_{n+1} & \sim \operatorname{coker} h_{n+1}
\end{aligned}
$$

(decorrentes da exatidão da sequência

$$
\left.\pi_{n+1} x \stackrel{h_{n+1}}{\longrightarrow} H_{n+1} \stackrel{\gamma_{n+1}}{\longrightarrow} \Gamma_{n} x \stackrel{\lambda_{n}}{\longrightarrow} \pi_{n} x \stackrel{h_{n}}{\longrightarrow} H_{n} x\right)
$$

o fato de kex $h_{n}$ e coker $h_{n+1}$ estarem em $c$ (itens iii) eiv) do teorema 6.1 .0$)$ e a proposição 3.2 .10 .

$$
\text { Deixamos os detalhes aos cuidados do leitor. }
$$





\section{BIBLIOGRAFIA}

[B] - Hans J.Baues, obstruction Theory, Lectures notes in Mathematics, n:628, Springer.

[B,K] - A.K.Bousfield, D.M. Kan, Homotopy Limits, Completions and Localization, Lectures notes in Mathematics, n? 304, springer.

[C,S] - H.Cartan, J.P.Serre, Espaces fibres et groupes d' homotopie, I.C.R. Acad.Sci.Paris 234, 1952, p. $288-290$.

[Ce] - E.Cech, Hoherdimensional Homotopiegruppen, Proc. Int. Congr. Mathematicians 3, 203 (Zurich, 1932).

[D] - E.Dror, A generalization of the Whitehead Theorem Lecture Notes in Mathematics, no 249, Springer, p. 13-22.

$\left[\mathrm{H}_{1}\right.$ - P.J.Hilton, An introduction to Homotopy Theory, Cambridge University Press.

[]$_{2}$ - P.J.Hilton, Remarks on the localization of nilpotent groups, Comm. Pure and App.Math. (1974). 
$\left[\mathrm{H}_{3}\right.$ - P.J.Hilton, Localization and cohomology of nilpotent groups, Math. Z. 132, p. 263-286.

$[\mathrm{H}]_{4}$ - P.J.Hilton, Nilpotent actions and nilpotent groups, Proc. Logic and Math. Conference, Springer, Lecture notes 450, (1975), 174-196.

$[\mathrm{H}]_{5}$ - P.J.Hilton, on G-spaces, Bol. Soc. Bras. Mat. 7, $(1976), 65-73$.

$[H, M, R]$ - P.J.Hilton, G.Mislin, J.Roitberg, Localization of nilpotent groups and spaces, Notas de Matemātica (55) North-Holland/American Elsevier.

$[H, R]_{1}$ - P.J.Hilton,J.Roitberg, Generalized C-theory and torsion phenomena in nilpotent spaces, Houston Journal of Mathematics 2 (1976), 525-559. $[\mathrm{H}, \mathrm{R}]_{2}$ - P.J.Hilton, J.Roitberg, On the Zeeman comparison theorem for the homology of quasi-nilpotent fibrations, Quarterly Journal of Mathematics, Vol. 27, n! 108, (1977), 433-444.

[H,W] - P.J.Hilton, S.Wylie, Homology Theory, Cambridge University Press, 1960.

[Hu] - W.Hurewicz, Beiträge zur Topologie der Deformatio nen, I, Hoherdiminsionale Homotopiegruppen, proc. Acad, von Wetenschappen, Amsterdam, 38, 112-119 (1935); II, Homotopie und Homologiegruppen, 38, 521-528 (1935); III, Klassen und Homologiegruppen von Abbildungen, 39, 117-126 
(1936); IV, Aspharische Rulume, 39, 215-224, (1936) .

[M] - W.S.Massey, Algebraic Topology: An Introduction, Harbrace College Mathematics Series, Harcourt, Brace \& World, Inc.

$[\mathrm{M}, \mathrm{T}]$ - R.E.Mosher, M.C.Tangora, Cohomology operations and Applications in Homotopy Theory, Harper \& Row Publishers.

[P] - H.Poincaré, Analysis situs, Jour. Ecole Polytech., (2) $1,1-121(1895)$.

[R] - J.S.Rose A course on group theory, Cambridge University Press.

$[S]_{1}$ - J.P.Serre, Homologie Singulière des espaces fibrés, Annals of Math. Vol. 54, n! 3, (1951) 425-505.

$[S]_{2}$ - J.P.Serre, Groupes d'Homotopie et classes de grou pes Abeliens, Annals of Math, vol. 58 nọ 2, (1953), $258-294$.

[Sp] - Spanier,E.H., Algebraic Topology, McGraw-Hil1 series in Higher Mathematics, 1966.

[Su] - D.Sulivan, Genetics of homotopy theory and the Adams conjecture, Annals of Math., 100 (1974),1-79.

[W] $]_{1}$ - J.H.C.Whitehead, Combinatorial Homotopy theory, I, II, Bull.Am.Math.Soc. 55 (1949), 213-245, 253-296

$[W]_{2}$ - J.H.C.Whitehead, A certain exact sequence, Ann. of Math., 52, (1950), 51-110. 